\title{
Noncommutative 3-colour scalar quantum field theory model in 2D
}

\author{
Alexander Hock ${ }^{\mathrm{a}}$, Raimar Wulkenhaar ${ }^{\mathrm{b}}$ \\ Mathematisches Institut der Westfälischen, Wilhelms-Universität, Einsteinstraße 62, 48149 Münster, Germany
}

Received: 30 April 2018 / Accepted: 2 July 2018 / Published online: 18 July 2018

(C) The Author(s) 2018

\begin{abstract}
We introduce the 3-colour noncommutative quantum field theory model in two dimensions. For this model we prove a generalised Ward-Takahashi identity, which is special to coloured noncommutative QFT models. It reduces to the usual Ward-Takahashi identity in a particular case. The Ward-Takahashi identity is used to simplify the SchwingerDyson equations for the 2-point function and the $N$-point function. The absence of any renormalisation conditions in the large $(\mathcal{N}, V)$-limit in 2D leads to a recursive integral equation for the 2-point function, which we solve perturbatively to sixth order in the coupling constant.
\end{abstract}

\section{Introduction}

Consider a hexagonal lattice with three different coloured links, where at each vertex all three links carry different colours. The mathematical problem of counting the number of colourings of a lattice with $N$ vertices was solved by Baxter [1]. A generalisation of this so-called 3-colour model as a Hermitian matrix model problem was introduced by Eynard and Kristjansen [2] and solved by Kostov [3]. Eynard and Kristjansen reduced the partition function (without external fields) to an integral over eigenvalues, which could be solved by saddle-point techniques.

Graphs in $\mathcal{N} \times \mathcal{N}$-matrix models are ribbon graphs on a Riemann surface. These ribbon graphs are dual to the triangulations of the corresponding surface. The large- $\mathcal{N}$ limit is dominated by planar graphs, corresponding to triangulations of the sphere. Two-dimensional quantum gravity can be formulated as a counting problem for triangulations of random surfaces, which leads to the connection between 2D quantum gravity and random matrices $[4,5]$.

Moreover, it was proved by Kontsevich [6] that the solution of an action of the form $\operatorname{tr}\left(E \cdot M^{2}+\frac{i}{6} M^{3}\right)$ with Her-

\footnotetext{
a e-mail: a_hock03@uni-muenster.de

b e-mail: raimar@math.uni-muenster.de
}

mitian matrices $M$ and external matrix $E$ can be mapped to a Hermitian matrix model with arbitrary potential. On the other hand the Kontsevich model proves Witten's conjecture about intersection numbers of stable cohomology classes on the moduli space of curves [7]. One particularly elegant solution technique reduced the partition function to an integral over the eigenvalues $x_{i}$ and observed that these integrals are unchanged under diffeomorphisms of $x_{i}$ generated by $x_{i}^{n+1} \frac{d}{d x_{i}}$. The corresponding Virasoro constraints all descend from a master constraint which was solved by Makeenko and Semenoff [8].

Matrix models gained renewed interest in a nonperturbative approach to quantum field theories on MoyalWeyl deformed noncommutative space [9,10]. These approaches use the matrix basis of the Moyal space and add a harmonic oscillator potential to the Laplacian [11]. The most established noncommutative quantum field theory is the $\Phi^{4}$-model [12], which is a candidate for an exactly solvable quantum field theory in 4D due to its vanishing $\beta$-function at all orders [13]. Recently all boundary sectors of noncommutative $\Phi^{3}$-model in $\{2,4,6\}$ dimensions were solved exactly in the large $(\mathcal{N}, V)$-limit $[14,15]$.

In this paper we will study the noncommutative 3-colour model as a quantum field theoretical model. Roughly speaking, it is the model solved by Kostov with an additional external dynamical field $E$ of linearly-spaced eigenvalues. Although it shares topologically some graphs with the noncommutative $\Phi^{3}$-model [14], it has more similarities to the $\Phi^{4}$-model [12] due to the absent 1-point function. For the large $(\mathcal{N}, V)$-limit a closed integral equation for the 2-point function will be derived. In the two-dimensional case the first-order loop correction has no UV divergence so that this 2D noncommutative 3-colour model needs no renormalisation in this limit. The closed integral equation defines a recursion formula for its perturbative expansion. Absence of any renormalisation makes this recursion easy. We are able to 
determine perturbatively the 2-point function up to the sixth order in the coupling constant.

The action of the noncommutative 3-colour model for real scalar fields $\phi^{a}$ with colour $a \in\{1,2,3\}$ is given by

$$
\begin{aligned}
S[\phi]:= & \frac{1}{8 \pi} \int_{\mathbb{R}^{2}} d x\left(\frac{1}{2} \sum_{a=1}^{3} \phi^{a}\left(-\Delta+\left\|2 \Theta^{-1} x\right\|^{2}+\mu^{2}\right) \phi^{a}\right. \\
& \left.+\frac{\lambda^{\prime}}{3} \sum_{a, b, c=1}^{3} \sigma_{a b c} \phi^{a} \star \phi^{b} \star \phi^{c}\right)(x),
\end{aligned}
$$

where $\sigma_{a b c}=1$ for $a \neq b \neq c \neq a$ and $\sigma_{a b c}=0$ else. Here $\lambda^{\prime} \in \mathbb{R}$ is the coupling constant, $\mu^{2}$ the mass squared and $\Delta$ the Laplacian, where independence of any colour is assumed. The Laplacian has non-compact resolvent, therefore the harmonic oscillator potential is added to achieve compactness. The Moyal $\star$-product is defined by

$$
\begin{aligned}
(f \star g)(x)= & \int_{\mathbb{R}^{2} \times \mathbb{R}^{2}} \frac{d y d k}{(2 \pi)^{2}} f\left(x+\frac{1}{2} \Theta k\right) \\
& \times g(x+y) e^{\mathrm{i}\langle k, y\rangle}, \quad f, g \in \mathcal{S}\left(\mathbb{R}^{2}\right),
\end{aligned}
$$

where $\Theta$ is a $2 \times 2$ skew-symmetric matrix with $\Theta_{12}=$ $-\Theta_{21}=: 4 V>0$.

The vector space $\mathcal{S}\left(\mathbb{R}^{2}\right)$ equipped with the Moyal $\star$ product has a matrix basis $f_{n m}(x)$, which is the 2-dimensional harmonic oscillator basis (independent of the harmonic oscillator term in (1.1), although equality of both frequencies reduces a tensor product to a matrix product). The formulation of the action in the matrix basis is obtained from the expansion

$\phi^{a}(x)=\sum_{n, m=0}^{\infty} \phi_{n m}^{a} f_{n m}(x)$, with $x \in \mathbb{R}^{2}$.

The matrix basis satisfies [16]

$\left(f_{n m} \star f_{k l}\right)(x)=\delta_{m k} f_{n l}(x), \quad \int_{\mathbb{R}^{2}} d x f_{n m}(x)=8 \pi V \delta_{n m}$.

Accordingly, the action in the matrix basis with an UV cut-off $\mathcal{N}$ is given by

$$
\begin{aligned}
S[\phi]= & V\left(\sum_{a=1}^{3} \sum_{n, m=0}^{\mathcal{N}} \frac{H_{n m}}{2} \phi_{n m}^{a} \phi_{m n}^{a}\right. \\
& \left.+\frac{\lambda^{\prime}}{3} \sum_{a, b, c=1}^{3} \sum_{n, m, l=0}^{\mathcal{N}} \sigma_{a b c} \phi_{n m}^{a} \phi_{m l}^{b} \phi_{l n}^{c}\right) \\
H_{n m}:= & E_{n}+E_{m}, \quad E_{m}=\frac{m}{V}+\frac{\mu^{2}}{2},
\end{aligned}
$$

where $\left(\phi_{n m}^{a}\right)$ are Hermitian matrices. The linear and discrete dependence of $E_{m}$ reflects the eigenvalue spectrum of the quantum-mechanical harmonic oscillator.

\section{Graph computation}

As a perturbative theory the noncommutative 3 -colour model can be expanded by graphs. In the large $(\mathcal{N}, V)$-limit taken later, all functions of genus $>0$ are suppressed, which is a well-known behaviour for matrix models. Therefore we will consider only planar ribbon graphs, but we admit a nontrivial boundary structure for which we next give an alternative description.

Let $\Gamma$ be a planar ribbon graph on $S^{2}$ consisting of vertices, edges and faces subject to the following conditions: It has two different vertices, black (internal) and white (external) vertices. The external vertex is also called boundary component. The number of white vertices is $B \geq 1$, and each white vertex has the valence $N_{\beta}$ for $\beta \in\{1, \ldots, B\}$. The edges have one of three different colours; they separate two faces. The black vertices are of valence three. At a black vertex all three colours must occur once. We require that every face has at most one external vertex, in which case it is called external. If the $\beta^{\text {th }}$ external vertex has valence $N_{\beta}$ it is a corner of $N_{\beta}$ external faces which are labelled by positive numbers $p_{1}^{\beta}, \ldots, p_{N_{\beta}}^{\beta}$. Let $a_{i}^{\beta}$ be the colour of the edge which ends at the $\beta^{\text {th }}$ external vertex and separates the faces labelled by $p_{i}^{\beta}$ and $p_{i+1}^{\beta}$, where $i \in\left\{1, \ldots, N_{\beta}\right\}$ and $N_{\beta}+1 \equiv 1$. Faces without an external vertex are called internal and are labelled by $q_{1}, \ldots, q_{L}$.

To every white vertex a weight 1 is associated and to every black vertex a weight $\lambda$. An edge is weighted by $\frac{1}{1+z_{1}+z_{2}}$ if $z_{1}$ and $z_{2}$ are the labels of the two faces separated by the edge.

To a graph $\Gamma$ we associate the function $\tilde{G}_{p_{1}^{1} \ldots p_{N_{1}}^{1}|\ldots| p_{1}^{B} \ldots p_{N_{B}}^{B}}^{a_{1}^{1} \ldots a_{N_{1}}^{1}|\ldots| a_{1}^{B} \ldots a_{N_{B}}^{B}}$ $(\Gamma)$ given by multiplication of all weights of vertices and faces of $\Gamma$ and with integration over the labels $q_{1}, \ldots, q_{L}$ of all internal faces from 0 to $\infty$. We consider two graphs $\Gamma, \Gamma^{\prime}$ as equivalent, $\Gamma \sim \Gamma^{\prime}$, if they are topologically the same and have the same labels $p_{1}^{1}, \ldots, p_{N_{1}}^{1}, \ldots, p_{1}^{B}, \ldots, p_{N_{B}}^{B}$ and $a_{1}^{1}, \ldots, a_{N_{1}}^{1}, \ldots, a_{1}^{B}, \ldots, a_{N_{B}}^{B}$, but different assignment of colours at internal edges. Such graphs $\Gamma \sim$ $\Gamma^{\prime}$ have the same amplitude $\tilde{G}_{p_{1}^{1} \ldots p_{N_{1}}^{1}|\cdots| p_{1}^{B} \ldots p_{N_{B}}^{B}}^{a_{1}^{1} \ldots a_{N_{1}}^{1}|\ldots| a^{B} \ldots a_{N_{B}}^{B}}(\Gamma)=$

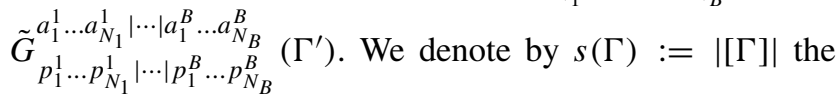
number of graphs equivalent to $\Gamma$.

For a fixed number $B$ of external vertices of valences $N_{1}, \ldots, N_{B}$, but arbitrary number of internal vertices, one can ask whether the sum over all possible planar graphs converges for sufficient small $|\lambda|$. This sum can formally be defined over all equivalence classes by 
$G_{p_{1}^{1} \ldots p_{N_{1}}^{1}|\cdots| p_{1}^{B} \ldots p_{N_{B}}^{B}}^{a_{1}^{1} \ldots a_{N_{1}}^{1}|\cdots| a_{1}^{B} \ldots a_{N_{B}}^{B}}:=\sum_{[\Gamma] \in \mathcal{G}_{\mathbf{p}}^{\mathbf{a}}} s(\Gamma) \tilde{G}_{p_{1}^{1} \ldots p_{N_{1}}^{1}|\cdots| p_{1}^{B} \ldots p_{N_{B}}^{B}}^{a_{1}^{1} \ldots a_{N_{1}}^{1}|\cdots| a_{1}^{B} \ldots a_{N_{B}}^{B}}(\Gamma), \quad \mathcal{Z}[J]:=\int\left(\prod_{a=1}^{3} \mathcal{D} \phi^{a}\right)$

where $\mathcal{G}_{\mathbf{p}}^{\mathbf{a}}$ is the set of equivalence classes of all planar graphs with $B$ external vertices, external edges of colour $a_{1}^{\beta}, \ldots, a_{N_{\beta}}^{\beta}$ and external faces labelled by $p_{1}^{\beta}, \ldots, p_{N_{\beta}}^{\beta}$ for all $\beta \in\{1, \ldots, B\}$. This sum can clearly be rearranged as a series in $\lambda$

$$
\begin{aligned}
& \times \exp \left(-S[\phi]+V \sum_{a=1}^{3} \sum_{n, m=0}^{\mathcal{N}} J_{n m}^{a} \phi_{m n}^{a}\right) \\
= & K \exp \left(-\frac{\lambda^{\prime}}{3 V^{2}} \sum_{a, b, c=1}^{3}\right. \\
& \left.\sum_{n, m, l=0}^{\mathcal{N}} \sigma_{a b c} \frac{\partial^{3}}{\partial J_{n m}^{a} \partial J_{m l}^{b} \partial J_{l n}^{c}}\right) \mathcal{Z}_{\text {free }}[J],
\end{aligned}
$$$$
G_{p_{1}^{1} \ldots p_{N_{1}}^{1}|\ldots| p_{1}^{B} \ldots p_{N_{B}}^{B}}^{a_{1}^{1} \ldots a_{N_{1}}^{1}|\cdots| a_{1}^{B} \ldots a_{N_{B}}^{B}}=: \sum_{n=0}^{\infty} \lambda^{n} G_{n, p_{1}^{1} \ldots p_{N_{1}}^{1}|\ldots| p_{1}^{B} \ldots p_{N_{B}}^{B}}^{a_{1}^{1} \ldots a_{N_{1}}^{1}|\ldots| a_{1}^{B} \ldots a_{N_{B}}^{B}}
$$

$\tilde{G}_{p_{1}^{1} \mid p_{1}^{2}}^{a_{1}^{1} \mid a^{2}}\left(\bigcirc^{p_{1}^{1}} \bigcirc\right)=\frac{\lambda^{2}}{\left(1+p_{1}^{1}+p_{1}^{2}\right)^{2}\left(1+2 p_{1}^{1}\right)\left(1+2 p_{1}^{2}\right)}$

$\begin{aligned} \tilde{G}_{p_{1}^{1} p_{2}^{1}}^{a_{1}^{1} a_{2}^{1}}\left(\lambda_{p_{2}^{1}}{ }^{q_{1}^{1}}\right. & =\frac{\lambda^{2}}{\left(1+p_{1}^{1}+p_{2}^{1}\right)^{2}} \int_{0}^{\infty} d q_{1} \frac{1}{\left(1+q_{1}+p_{1}^{1}\right)\left(1+q_{1}+p_{2}^{1}\right)} \\ & =\lambda^{2} \frac{\log \left(1+p_{1}^{1}\right)-\log \left(1+p_{2}^{1}\right)}{\left(1+p_{1}^{1}+p_{2}^{1}\right)^{2}\left(p_{1}^{1}-p_{2}^{1}\right)}\end{aligned}$

$\tilde{G}_{p_{1}^{1} p_{2}^{1} p_{3}^{1}}^{a_{1}^{1} a_{2}^{1} a_{3}^{1}}(\underbrace{q_{1}}_{p_{1}^{1}}{ }_{p_{2}^{1}}^{1} p_{p_{3}^{1}})$

$=\frac{\lambda^{3}}{\left(1+p_{1}^{1}+p_{2}^{1}\right)\left(1+p_{2}^{1}+p_{3}^{1}\right)\left(1+p_{1}^{1}+p_{3}^{1}\right)}$

$\times \int_{0}^{\infty} d q_{1} \frac{1}{\left(1+q_{1}+p_{1}^{1}\right)\left(1+q_{1}+p_{2}^{1}\right)\left(1+q_{1}+p_{3}^{1}\right)}$

$=\lambda^{3} \frac{\frac{\log \left(1+p_{1}^{1}\right)}{\left(p_{1}^{1}-p_{2}^{1}\right)\left(p_{3}^{1}-p_{1}^{1}\right)}+\frac{\log \left(1+p_{2}^{1}\right)}{\left(p_{2}^{1}-p_{1}^{1}\right)\left(p_{3}^{1}-p_{2}^{1}\right)}+\frac{\log \left(1+p_{3}^{1}\right)}{\left(p_{3}^{1}-p_{1}^{1}\right)\left(p_{2}^{1}-p_{3}^{1}\right)}}{\left(1+p_{1}^{1}+p_{2}^{1}\right)\left(1+p_{2}^{1}+p_{3}^{1}\right)\left(1+p_{1}^{1}+p_{3}^{1}\right)}$

The equivalence class corresponding to the second example contains two elements, so we obtain for $a=a_{1}^{1}=a_{2}^{1}$

$G_{2, p_{1} p_{2}}^{a a}=\frac{2 \log \left(\frac{1+p_{1}}{1+p_{2}}\right)}{\left(1+p_{1}+p_{2}\right)^{2}\left(p_{1}-p_{2}\right)}$.

\section{Partition function and correlation function}

In the following we demonstrate the techniques to determine correlation functions from the partition function. The partition function $\mathcal{Z}[\mathrm{J}]$ of the noncommutative 3-colour model with external Hermitian matrices $\left(J_{n m}^{a}\right)$ and $a \in\{1,2,3\}$ is formally defined by

$$
\begin{aligned}
\mathcal{Z}_{\text {free }}[J] & :=\exp \left(\sum_{a=1}^{3} \sum_{n, m=0}^{\mathcal{N}} \frac{V}{2 H_{n m}} J_{n m}^{a} J_{m n}^{a}\right), \\
K:= & \int\left(\prod_{a=1}^{3} \mathcal{D} \phi^{a}\right) \\
& \times \exp \left(-\sum_{a=1}^{3} \sum_{n, m=0}^{\mathcal{N}} \frac{V H_{n m}}{2} \phi_{n m}^{a} \phi_{m n}^{a}\right) .
\end{aligned}
$$

The logarithm of $\mathcal{Z}[J]$ will be expanded into a series of moments with different number $B$ of boundary components. These moments are called correlation functions, which not necessarily correspond to planar graphs. The sources are cyclic within every boundary $\beta \in\{1, \ldots, B\}$. For simpli- 
fication we use the notation $\mathbb{J}_{p_{1}^{\beta} \ldots p_{N_{\beta}}^{\beta}}^{a_{1}^{\beta} \ldots a_{N_{\beta}}^{\beta}}:=\prod_{i=1}^{N_{\beta}} J_{p_{i}^{\beta} p_{i+1}^{\beta}}^{a_{i}^{\beta}}$ with $N_{\beta}+1 \equiv 1$. The correlation functions are then defined by

$$
\begin{aligned}
\log \frac{\mathcal{Z}[J]}{\mathcal{Z}[0]} & =: \sum_{B=1}^{\infty} \sum_{1 \leq N_{1} \leq \cdots \leq N_{B}}^{\infty} \sum_{p_{1}^{1}, \ldots, p_{N_{B}}^{B}=0}^{\mathcal{N}} \\
& \times \sum_{a_{1}^{1}, \ldots, a_{N_{B}}^{B}=1}^{3} V^{2-B} \frac{G_{\left|p_{1}^{1} \ldots p_{N_{1}}^{1}\right| \ldots\left|p_{1}^{B} \ldots p_{N_{B}}^{B}\right|}^{a_{1}^{1} \ldots a_{N_{1}}^{1}|\cdots| a_{1}^{B} \ldots a_{N_{B}}^{B}}}{S_{\left(N_{1}, \ldots, N_{B}\right)}^{B}} \prod_{\beta=1}^{\mathbb{J}_{p_{1}^{\beta} \ldots a_{N_{\beta}}^{\beta}}^{\beta}} \frac{p_{N_{\beta}}^{\beta}}{N_{\beta}} .
\end{aligned}
$$

If we regroup identical numbers $N_{\beta}$ by $\left(N_{1}, \ldots, N_{B}\right)=$ $(\underbrace{N_{1}^{\prime}, \ldots, N_{1}^{\prime}}_{v_{1}}, \ldots, \underbrace{N_{s}^{\prime}, \ldots, N_{s}^{\prime}}_{v_{s}})$, the symmetry factor is then defined by $S_{\left(N_{1}, \ldots, N_{B}\right)}:=\prod_{i=1}^{s} v_{i}$ !, due to the symmetry of boundaries with the same valence. The expansion coefficients $G_{\left|p_{1}^{1} \ldots p_{N_{1}}^{1}\right| \ldots\left|p_{1}^{B} \ldots p_{N_{B}}^{B}\right|}^{a_{1}^{1} \ldots a_{N_{1}}^{1}|\cdots| a_{1}^{B} \ldots a_{N_{B}}^{B}}$ are called $\left(N_{1}+\cdots+N_{B}\right)$-point functions. For the time being, the factor $V^{2-B}$ in (3.3) is a matter of convention; it could be absorbed in $G$. However, as known from [14] precisely this convention leads to equations for the $\left(N_{1}+\cdots+N_{B}\right)$-point functions which all have a well-defined large $(\mathcal{N}, V)$-limit.

Due to the vanishing 1-point function for the 3-colour model, the partition function can be expanded with (3.3) to

$$
\begin{aligned}
\frac{\mathcal{Z}[J]}{\mathcal{Z}[0]}= & 1+\sum_{a, b=1}^{3} \sum_{n, m=0}^{\mathcal{N}}\left(\frac{V}{2} G_{|n m|}^{a b} \mathbb{J}_{n m}^{a b}+\frac{1}{2} G_{|n| m \mid}^{a \mid b} \mathbb{J}_{n}^{a} \mathbb{J}_{m}^{b}\right) \\
& +\sum_{a, b, c=1}^{3} \sum_{n, m, l=0}^{\mathcal{N}}\left(\frac{V}{3} G_{|n m l|}^{a b c} \mathbb{J}_{n m l}^{a b c}+\frac{1}{2} G_{|n| m l \mid}^{a \mid b c} \mathbb{J}_{n}^{a} \mathbb{J}_{m l}^{b c}\right. \\
& \left.+\frac{1}{6 V} G_{|n| m|l|}^{a|b| c} \mathbb{J}_{n}^{a} \mathbb{J}_{m}^{b} \mathbb{J}_{l}^{c}\right) \\
& +\sum_{a, b, c, d=1}^{3} \sum_{n, m, l, p=0}^{\mathcal{N}}\left(\frac{V}{4} G_{|n m l p|}^{a b c d} \mathbb{J}_{n m l p}^{a b c d}+\frac{1}{3} G_{|n| m l p \mid}^{a \mid b c d} \mathbb{J}_{n}^{a} \mathbb{J}_{m l p}^{b c d}\right. \\
& +\left(\frac{1}{8} G_{|n m| l|p|}^{a b \mid c d}+\frac{V^{2}}{8} G_{|n m|}^{a b} G_{|l p|}^{c d}\right) \mathbb{J}_{n m}^{a b} \mathbb{J}_{l p}^{c d} \\
& +\left(\frac{1}{4 V} G_{|n| m|l p|}^{a|b| c d}+\frac{V}{4} G_{|n| m \mid}^{a \mid b} G_{|l p|}^{c d}\right) \mathbb{J}_{n}^{a} \mathbb{J}_{m}^{b} \mathbb{J}_{l p}^{c d} \\
& \left.+\left(\frac{1}{24 V^{2}} G_{|n| m|l| p \mid}^{a|b| c \mid d}+\frac{1}{8} G_{|n| m \mid}^{a \mid b} G_{|l| p \mid}^{c \mid d}\right) \mathbb{J}_{n}^{a} \mathbb{J}_{m}^{b} \mathbb{J}_{l}^{c} \mathbb{J}_{p}^{d}\right)+\cdots
\end{aligned}
$$

The calculation rule for later purpose is

$\frac{\partial}{\partial J_{p_{1} p_{2}}^{a}} J_{p_{3} p_{4}}^{b}=\delta_{a b} \delta_{p_{1} p_{3}} \delta_{p_{2} p_{4}}+J_{p_{3} p_{4}}^{b} \frac{\partial}{\partial J_{p_{1} p_{2}}^{a}}$.

\section{Ward-Takahashi identity}

The Ward-Takahashi identity is obtained by the requirement of invariance of $\mathcal{Z}[J]$ under inner automorphisms. For a colour model we choose a transformation as follows: $\phi^{a} \mapsto\left(\phi^{a}\right)^{\prime}=U^{\dagger} \phi^{a} U$ for $U \in \mathrm{U}(\mathcal{N})$ for one colour $a \in\{1,2,3\}$. The Ward-Takahashi identity following from this transformation $[12,13]$ for $p_{1} \neq p_{2}$ is given by

$$
\begin{aligned}
\sum_{m=0}^{\mathcal{N}} & \frac{\partial^{2}}{\partial J_{p_{1} m}^{a} \partial J_{m p_{2}}^{a}} \mathcal{Z}[J]+\frac{V}{E_{p_{1}}-E_{p_{2}}} \\
& \times \sum_{m=0}^{\mathcal{N}}\left(J_{p_{2} m}^{a} \frac{\partial}{\partial J_{p_{1} m}^{a}}-J_{m p_{1}}^{a} \frac{\partial}{\partial J_{m p_{2}}^{a}}\right) \mathcal{Z}[J] \\
= & \frac{\lambda^{\prime}}{V\left(E_{p_{1}}-E_{p_{2}}\right)} \sum_{m, n=0}^{\mathcal{N}} \\
& \times \sum_{b, c=1}^{3} \sigma_{a b c}\left(\frac{\partial^{3}}{\partial J_{p_{1} m}^{a} \partial J_{m n}^{b} \partial J_{n p_{2}}^{c}}-\frac{\partial^{3}}{\partial J_{p_{1} m}^{b} \partial J_{m n}^{c} \partial J_{n p_{2}}^{a}}\right) \mathcal{Z}[J] .
\end{aligned}
$$

The interaction terms are certainly not invariant under the transformation of only one colour. However, the sum over all colours in (4.1) gives

$$
\begin{aligned}
& \sum_{a=1}^{3} \sum_{m=0}^{\mathcal{N}} \frac{\partial^{2}}{\partial J_{p_{1} m}^{a} \partial J_{m p_{2}}^{a}} \mathcal{Z}[J]=\frac{V}{\left(E_{p_{1}}-E_{p_{2}}\right)} \sum_{a=1}^{3} \\
& \quad \times \sum_{m=0}^{\mathcal{N}}\left(J_{m p_{1}}^{a} \frac{\partial}{\partial J_{m p_{2}}^{a}}-J_{p_{2} m}^{a} \frac{\partial}{\partial J_{p_{1} m}^{a}}\right) \mathcal{Z}[J]
\end{aligned}
$$

which has the usual form of a Ward-Takahashi identity. Equation (4.2) shows that the interaction term is invariant under the simultaneous transformation of all three colours.

A crucial rôle plays a more general identity:

Proposition 1 Let $p_{1} \neq p_{2}$. The generalised WardTakahashi identity for the 3-colour matrix model with an external field $E$ is

$$
\begin{aligned}
\sum_{m=0}^{\mathcal{N}} & \frac{\partial^{2}}{\partial J_{p_{1} m}^{a} \partial J_{m p_{2}}^{b}} \mathcal{Z}[J]+\frac{V}{E_{p_{1}}-E_{p_{2}}} \\
& \times \sum_{m=0}^{\mathcal{N}}\left(J_{p_{2} m}^{b} \frac{\partial}{\partial J_{p_{1} m}^{a}}-J_{m p_{1}}^{a} \frac{\partial}{\partial J_{m p_{2}}^{b}}\right) \mathcal{Z}[J] \\
= & \frac{\lambda^{\prime}}{V\left(E_{p_{1}}-E_{p_{2}}\right)} \sum_{m, n=0}^{\mathcal{N}} \sum_{c, d=1}^{3}\left(\sigma_{b c d} \frac{\partial^{3}}{\partial J_{p_{1} m}^{a} \partial J_{m n}^{c} \partial J_{n p_{2}}^{d}}\right. \\
& \left.-\sigma_{a c d} \frac{\partial^{3}}{\partial J_{p_{1} m}^{c} \partial J_{m n}^{d} \partial J_{n p_{2}}^{b}}\right) \mathcal{Z}[J] .
\end{aligned}
$$


Proof Let $S_{i n t}[\phi]=V \frac{\lambda^{\prime}}{3} \sum_{a, b, c=1}^{3} \sum_{n, m, l=0}^{\mathcal{N}} \sigma_{a b c} \phi_{n m}^{a} \phi_{m l}^{b} \phi_{l n}^{c}$ be the interaction term of the action. Direct computation gives then

$$
\begin{aligned}
\frac{E_{p_{1}}-E_{p_{2}}}{V} \sum_{m=0}^{\mathcal{N}} \frac{\partial^{2}}{\partial J_{p_{1} m}^{a} \partial J_{m p_{2}}^{b}} \mathcal{Z}[J] \\
=\frac{1}{V} \sum_{m=0}^{\mathcal{N}} \frac{\partial^{2}}{\partial J_{p_{1} m}^{a} \partial J_{m p_{2}}^{b}}\left(\left(E_{p_{1}}+E_{m}\right)-\left(E_{m}+E_{p_{2}}\right)\right) \mathcal{Z}[J] \\
=K \sum_{m=0}^{\mathcal{N}}\left\{\frac{\partial}{\partial J_{m p_{2}}^{b}} \exp \left(-S_{i n t}\left[\frac{1}{V} \frac{\partial}{\partial J}\right]\right) J_{m p_{1}}^{a}\right. \\
\left.\quad-\frac{\partial}{\partial J_{p_{1} m}^{a}} \exp \left(-S_{i n t}\left[\frac{1}{V} \frac{\partial}{\partial J}\right]\right) J_{p_{2} m}^{b}\right\} \mathcal{Z}_{\text {free }}[J] \\
=\sum_{m=0}^{\mathcal{N}}\left(J_{m p_{1}}^{a} \frac{\partial}{\partial J_{m p_{2}}^{b}}-J_{p_{2} m}^{b} \frac{\partial}{\partial J_{p_{1} m}^{a}}\right) \mathcal{Z}[J] \\
\quad-\frac{\lambda^{\prime}}{V^{2}} \sum_{m, n=0}^{\mathcal{N}} \sum_{c, d=1}^{3}\left(\sigma_{a c d} \frac{\partial^{3}}{\partial J_{p_{1} n}^{c} \partial J_{n m}^{d} \partial J_{m p_{2}}^{b}}\right. \\
\left.\quad-\sigma_{b c d} \frac{\partial^{3}}{\partial J_{p_{1} m}^{a} \partial J_{m n}^{c} \partial J_{n p_{2}}^{d}}\right) \mathcal{Z}[J] .
\end{aligned}
$$

We have used the second form of $\mathcal{Z}[J]$ in (3.1) and the Leibniz rule in the last step. Technically one expands the exponential function and resums after using the Leibniz rule. Since $E_{p_{1}} \neq E_{p_{2}}$ the proof is finished.

Equation (4.1) is a special case of Proposition 1 by setting $b=a$. The derivation of both identities is completely different. Proposition 1 can not be obtained by a symmetry transformation of only one colour due to the discrete mixing of the colours if $a \neq b$. Applying the procedure of the proof of Proposition 1, it is also possible to derive the usual Ward-Takahashi identity even in other models.

For later purpose we combine two identities to get a more useful expression:

Lemma 1 Let a be fixed and $p_{1} \neq p_{2}$, then it follows

$$
\begin{aligned}
& \sum_{b, c=1}^{3} \sum_{m=0}^{\mathcal{N}} \sigma_{a b c} \frac{\partial^{2}}{\partial J_{p_{1} m}^{b} \partial J_{m p_{2}}^{c}} \mathcal{Z}[J] \\
& =\frac{V}{E_{p_{1}}-E_{p_{2}}}\left[\sum_{b, c=1}^{3} \sigma_{a b c} \sum_{m=0}^{\mathcal{N}}\left(J_{m p_{1}}^{b} \frac{\partial}{\partial J_{m p_{2}}^{c}}-J_{p_{2} m}^{c} \frac{\partial}{\partial J_{p_{1} m}^{b}}\right)\right. \\
& \quad+\frac{\lambda^{\prime}}{V^{2}} \sum_{b=1}^{3}\left\{\sum_{m=0}^{\mathcal{N}}\left(\frac{\partial^{3}}{\partial J_{p_{1} m}^{b} \partial J_{m p_{1}}^{b} \partial J_{p_{1} p_{2}}^{a}}-\frac{\partial^{3}}{\partial J_{p_{1} p_{2}}^{a} \partial J_{p_{2} m}^{b} \partial J_{m p_{2}}^{b}}\right)\right. \\
& \quad+\sum_{\substack{m, n=0 \\
n \neq p_{1}}}^{\mathcal{N}} \frac{V}{E_{p_{1}}-E_{n}} \frac{\partial}{\partial J_{n p_{2}}^{a}}\left(J_{m p_{1}}^{b} \frac{\partial}{\partial J_{m n}^{b}}-J_{n m}^{b} \frac{\partial}{\partial J_{p_{1} m}^{b}}\right)
\end{aligned}
$$

$$
\left.\left.-\sum_{\substack{m, n=0 \\ n \neq p_{2}}}^{\mathcal{N}} \frac{V}{E_{p_{2}}-E_{n}} \frac{\partial}{\partial J_{p_{1} n}^{a}}\left(J_{p_{2} m}^{b} \frac{\partial}{\partial J_{n m}^{b}}-J_{m n}^{b} \frac{\partial}{\partial J_{m p_{2}}^{b}}\right)\right\}\right] \mathcal{Z}[J]
$$

Proof Inserting Proposition 1 for the LHS yields

$$
\begin{aligned}
\sum_{b, c} & \sum_{m=0}^{\mathcal{N}} \sigma_{a b c} \frac{\partial^{2}}{\partial J_{p_{1} m}^{b} \partial J_{m p_{2}}^{c}} \mathcal{Z}[J] \\
= & \frac{V}{E_{p_{1}}-E_{p_{2}}} \sum_{b, c=1}^{3} \sigma_{a b c} \sum_{m=0}^{\mathcal{N}}\left(J_{m p_{1}}^{b} \frac{\partial}{\partial J_{m p_{2}}^{c}}-J_{p_{2} m}^{c} \frac{\partial}{\partial J_{p_{1} m}^{b}}\right) \mathcal{Z}[J] \\
& +\frac{\lambda^{\prime}}{V\left(E_{p_{1}}-E_{p_{2}}\right)} \sum_{m, n=0}^{\mathcal{N}} \sum_{b, c, d, e=1}^{3} \sigma_{a b c}\left(\sigma_{c d e} \frac{\partial^{3}}{\partial J_{p_{1} m}^{b} \partial J_{m n}^{d} \partial J_{n p_{2}}^{e}}\right. \\
& \left.-\sigma_{b d e} \frac{\partial^{3}}{\partial J_{p_{1} m}^{d} \partial J_{m n}^{e} \partial J_{n p_{2}}^{c}}\right) \mathcal{Z}[J] .
\end{aligned}
$$

By the sum over the colours $b, c, d, e$, we obtain for the multiplication of two $\sigma$ 's with one common index

$\sigma_{a b c} \sigma_{c d e}=\sigma_{a b c}\left(\delta_{a d} \delta_{b e}+\delta_{a e} \delta_{b d}\right)$

$\sigma_{a b c} \sigma_{b d e}=\sigma_{a b c}\left(\delta_{a d} \delta_{c e}+\delta_{a e} \delta_{c d}\right)$.

Therefore, the last line in (4.3) gives

$$
\begin{aligned}
& \frac{\lambda^{\prime}}{V\left(E_{p_{1}}-E_{p_{2}}\right)} \sum_{m, n=0}^{\mathcal{N}} \sum_{b, c=1}^{3} \\
& \times \sigma_{a b c}\left(\frac{\partial^{3}}{\partial J_{p_{1} m}^{b} \partial J_{m n}^{a} \partial J_{n p_{2}}^{b}}+\frac{\partial^{3}}{\partial J_{p_{1} m}^{b} \partial J_{m n}^{b} \partial J_{n p_{2}}^{a}}\right. \\
& \left.-\frac{\partial^{3}}{\partial J_{p_{1} m}^{a} \partial J_{m n}^{c} \partial J_{n p_{2}}^{c}}-\frac{\partial^{3}}{\partial J_{p_{1} m}^{c} \partial J_{m n}^{a} \partial J_{n p_{2}}^{c}}\right) \mathcal{Z}[J] .
\end{aligned}
$$

The first and the last term in parentheses vanish because of the total symmetry of $\sigma_{a b c}$. Adding $0=$ $\left(\frac{\partial^{3}}{\partial J_{p_{1} m}^{a} \partial J_{m n}^{a} \partial J_{n p_{2}}^{a}}-\frac{\partial^{3}}{\partial J_{p_{1} m}^{a} \partial J_{m n}^{a} \partial J_{n p_{2}}^{a}}\right) \mathcal{Z}[J]$ and renaming the indices, (4.4) can be rewritten to

$$
\frac{\lambda^{\prime}}{V\left(E_{p_{1}}-E_{p_{2}}\right)} \sum_{m, n=0}^{\mathcal{N}} \sum_{b=1}^{3}\left(\frac{\partial^{3}}{\partial J_{p_{1} m}^{b} \partial J_{m n}^{b} \partial J_{n p_{2}}^{a}}-\frac{\partial^{3}}{\partial J_{p_{1} m}^{a} \partial J_{m n}^{b} \partial J_{n p_{2}}^{b}}\right) \mathcal{Z}[J] .
$$

Inserting (4.2) for $n \neq p_{1}$ in the first and $m \neq p_{2}$ in the second term gives after renaming indices finally

$$
\begin{aligned}
& \frac{\lambda^{\prime}}{V\left(E_{p_{1}}-E_{p_{2}}\right)} \sum_{b=1}^{3}\left\{\sum_{m=0}^{\mathcal{N}}\left(\frac{\partial^{3}}{\partial J_{p_{1} m}^{b} \partial J_{m p_{1}}^{b} \partial J_{p_{1} p_{2}}^{a}}-\frac{\partial^{3}}{\partial J_{p_{1} p_{2}}^{a} \partial J_{p_{2} m}^{b} \partial J_{m p_{2}}^{b}}\right)\right. \\
& +\sum_{\substack{m, n=0 \\
n \neq p_{1}}}^{\mathcal{N}} \frac{V}{E_{p_{1}}-E_{n}} \frac{\partial}{\partial J_{n p_{2}}^{a}}\left(J_{m p_{1}}^{b} \frac{\partial}{\partial J_{m n}^{b}}-J_{n m}^{b} \frac{\partial}{\partial J_{p_{1} m}^{b}}\right)
\end{aligned}
$$




$$
\left.-\sum_{\substack{m, n=0 \\ n \neq p_{2}}}^{\mathcal{N}} \frac{V}{E_{p_{2}}-E_{n}} \frac{\partial}{\partial J_{p_{1} n}^{a}}\left(J_{p_{2} m}^{b} \frac{\partial}{\partial J_{n m}^{b}}-J_{m n}^{b} \frac{\partial}{\partial J_{m p_{2}}^{b}}\right)\right\} \mathcal{Z}[J] .
$$

The identity follows by combining (4.3) and (4.5).

\section{Schwinger-Dyson equations for $B=1$}

\subsection{For matrix basis}

In this section we derive the Schwinger-Dyson equations with the help of Ward-Takahashi identity.

Proposition 2 The Schwinger-Dyson equation for the 2point function in the 3-colour matrix model with an external field $E$ is for $p_{1} \neq p_{2}$ given by

$$
\begin{aligned}
& G_{\left|p_{1} p_{2}\right|}^{a a}=\frac{1}{H_{p_{1} p_{2}}}+\frac{\lambda^{\prime 2}}{\left(E_{p_{1}}^{2}-E_{p_{2}}^{2}\right) V} \\
& \times\left[\sum _ { m = 0 } ^ { \mathcal { N } } \sum _ { b = 1 } ^ { 3 } \left(G_{\left|p_{1} p_{2}\right|}^{a a}\left(G_{\left|p_{2} m\right|}^{b b}-G_{\left|p_{1} m\right|}^{b b}\right)\right.\right. \\
& \left.+\frac{1}{V}\left(G_{\left|p_{2} p_{1} p_{2} m\right|}^{a a b b}-G_{\left|p_{1} p_{2} p_{1} m\right|}^{a a b b}\right)\right) \\
& +\sum_{b=1}^{3} \frac{1}{V^{2}}\left(\sum_{m=0}^{\mathcal{N}}\left(G_{\left|p_{2} p_{1}\right| p_{2} m \mid}^{a a \mid b b}-G_{\left|p_{1} p_{2}\right| p_{1} m \mid}^{a a \mid b b}\right)\right. \\
& \left.+\left(G_{\left|p_{2}\right| p_{2} p_{2} p_{1} \mid}^{b \mid b a a}-G_{\left|p_{1}\right| p_{1} p_{1} p_{2} \mid}^{b \mid b a a}\right)\right) \\
& +\sum_{b=1}^{3}\left(\frac{1}{V^{3}}\left(G_{\left|p_{2}\right| p_{2}\left|p_{2} p_{1}\right|}^{b|b| a a}-G_{\left|p_{1}\right| p_{1}\left|p_{1} p_{2}\right|}^{b|b| a a}\right)\right. \\
& \left.+\frac{1}{V} G_{\left|p_{1} p_{2}\right|}^{a a}\left(G_{\left|p_{2}\right| p_{2} \mid}^{b \mid b}-G_{\left|p_{1}\right| p_{1} \mid}^{b \mid b}\right)\right) \\
& +\sum_{\substack{m=0 \\
m \neq p_{2}}}^{\mathcal{N}} \frac{G_{\left|p_{1} m\right|}^{a a}-G_{\left|p_{1} p_{2}\right|}^{a a}}{E_{p_{2}}-E_{m}}-\sum_{\substack{m=0 \\
m \neq p_{1}}}^{\mathcal{N}} \frac{G_{\left|p_{1} p_{2}\right|}^{a a}-G_{\left|p_{2} m\right|}^{a a}}{E_{m}-E_{p_{1}}} \\
& \left.+\frac{1}{V} \frac{G_{\left|p_{1}\right| p_{1} \mid}^{a \mid a}-2 G_{\left|p_{1}\right| p_{2} \mid}^{a \mid a}+G_{\left|p_{2}\right| p_{2} \mid}^{a \mid a}}{E_{p_{2}}-E_{p_{1}}}\right] .
\end{aligned}
$$

Proof Assuming $p_{1} \neq p_{2}$ the 2-point function is given via definition (3.3) and expansion (3.4). Using (3.1) leads to

$$
\begin{aligned}
G_{\left|p_{1} p_{2}\right|}^{a a}= & \left.\frac{1}{V} \frac{\partial^{2}}{\partial J_{p_{1} p_{2}}^{a} \partial J_{p_{2} p_{1}}^{a}} \log \mathcal{Z}[J]\right|_{J=0} \\
= & \left.\frac{1}{V \mathcal{Z}[0]} \frac{\partial^{2}}{\partial J_{p_{1} p_{2}}^{a} \partial J_{p_{2} p_{1}}^{a}} \mathcal{Z}[J]\right|_{J=0} \\
= & \frac{K}{H_{p_{1} p_{2}} \mathcal{Z}[0]} \frac{\partial}{\partial J_{p_{2} p_{1}}^{a}} \\
& \times\left.\exp \left(-S_{i n t}\left[\frac{1}{V} \frac{\partial}{\partial J}\right]\right) J_{p_{2} p_{1}}^{a} \mathcal{Z}_{\text {free }}[J]\right|_{J=0}
\end{aligned}
$$

$$
\begin{aligned}
= & \frac{1}{H_{p_{1} p_{2}}}-\frac{\lambda^{\prime}}{H_{p_{1} p_{2}} \mathcal{Z}[0] V^{2}} \frac{\partial}{\partial J_{p_{2} p_{1}}^{a}} \sum_{b, c=1}^{3} \\
& \times\left.\sum_{m=0}^{\mathcal{N}} \sigma_{a b c} \frac{\partial^{2}}{\partial J_{p_{1} m}^{b} \partial J_{m p_{2}}^{c}} \mathcal{Z}[J]\right|_{J=0} .
\end{aligned}
$$

Inserting the expansion of (3.4) would give the SchwingerDyson equation between the 2-point and 3-point function. At first sight the application of Lemma 1 seems to make the equation more complicated. However, it yields a better behaviour in the large $(\mathcal{N}, V)$-limit. The first term on the RHS of the equation of Lemma 1 vanishes by setting $J$ to zero. Therefore, we obtain

$$
\begin{aligned}
=\frac{1}{H_{p_{1} p_{2}}}-\frac{\lambda^{\prime 2}}{\left(E_{p_{1}}^{2}-E_{p_{2}}^{2}\right) \mathcal{Z}[0] V^{3}} & \left\{\sum _ { b = 1 } ^ { 3 } \sum _ { m = 0 } ^ { \mathcal { N } } \left(\frac{\partial^{4}}{\partial J_{p_{1} m}^{b} \partial J_{m p_{1}}^{b} \partial J_{p_{1} p_{2}}^{a} \partial J_{p_{2} p_{1}}^{a}}\right.\right. \\
& \left.-\frac{\partial^{4}}{\partial J_{p_{2} p_{1}}^{a} \partial J_{p_{1} p_{2}}^{a} \partial J_{p_{2} m}^{b} \partial J_{m p_{2}}^{b}}\right)\left.\mathcal{Z}[J]\right|_{J=0} \\
& +\sum_{\substack{m, n=0 \\
n \neq p_{1}}}^{\mathcal{N}} \frac{V}{E_{p_{1}}-E_{n}} \frac{\partial^{2}}{\partial J_{p_{2} p_{1}}^{a} \partial J_{n p_{2}}^{a}}\left(J_{m p_{1}}^{a} \frac{\partial}{\partial J_{m n}^{a}}\right. \\
& \left.-J_{n m}^{a} \frac{\partial}{\partial J_{p_{1} m}^{a}}\right)\left.\mathcal{Z}[J]\right|_{J=0} \\
& -\sum_{\substack{m, n=0 \\
n \neq p_{2}}}^{\mathcal{N}} \frac{V}{E_{p_{2}}-E_{n}} \frac{\partial^{2}}{\partial J_{p_{2} p_{1}}^{a} \partial J_{p_{1} n}^{a}}\left(J_{p_{2} m}^{a} \frac{\partial}{\partial J_{n m}^{a}}\right. \\
& \left.\left.-J_{m n}^{a} \frac{\partial}{\partial J_{m p_{2}}^{a}}\right)\left.\mathcal{Z}[J]\right|_{J=0}\right\},
\end{aligned}
$$

where $H_{p_{1} p_{2}}\left(E_{p_{1}}-E_{p_{2}}\right)=\left(E_{p_{1}}^{2}-E_{p_{2}}^{2}\right)$ has been used and the fact that in the last two lines only colour $a$ survives. By taking $p_{1} \neq p_{2}$ into account and $J=0$ gives with the Leibniz rule

$$
\begin{gathered}
=\frac{1}{H_{p_{1} p_{2}}}-\frac{\lambda^{\prime 2}}{\left(E_{p_{1}}^{2}-E_{p_{2}}^{2}\right) \mathcal{Z}[0] V^{3}} \\
\quad \times\left\{\left.\sum_{b=1}^{3} \sum_{m=0}^{\mathcal{N}}\left(\frac{\partial^{4}}{\partial J_{p_{1} m}^{b} \partial J_{m p_{1}}^{b} \partial J_{p_{1} p_{2}}^{a} \partial J_{p_{2} p_{1}}^{a}}\right) \mathcal{Z}[J]\right|_{J=0}\right. \\
\left.\quad-\frac{\partial^{4}}{\partial J_{p_{2} p_{1}}^{a} \partial J_{p_{1} p_{2}}^{a} \partial J_{p_{2} m}^{b} \partial J_{m p_{2}}^{b}}\right) \\
+\sum_{\substack{m=0 \\
m \neq p_{1}}}^{\mathcal{N}} \frac{V}{E_{p_{1}}-E_{m}}\left(\frac{\partial^{2}}{\partial J_{m p_{2}}^{a} \partial J_{p_{2} m}^{a}}\right.
\end{gathered}
$$




$$
\begin{aligned}
& \left.-\frac{\partial^{2}}{\partial J_{p_{1} p_{2}}^{a} \partial J_{p_{2} p_{1}}^{a}}\right)\left.\mathcal{Z}[J]\right|_{J=0} \\
& -\sum_{\substack{m=0 \\
m \neq p_{2}}}^{\mathcal{N}} \frac{V}{E_{p_{2}}-E_{m}}\left(\frac{\partial^{2}}{\partial J_{m p_{1}}^{a} \partial J_{p_{1} m}^{a}}\right. \\
& \left.-\frac{\partial^{2}}{\partial J_{p_{1} p_{2}}^{a} \partial J_{p_{2} p_{1}}^{a}}\right)\left.\mathcal{Z}[J]\right|_{J=0} \\
& +\frac{V}{E_{p_{2}}-E_{p_{1}}}\left(\frac{\partial^{2}}{\partial J_{p_{2} p_{2}}^{a} \partial J_{p_{1} p_{1}}^{a}}\right. \\
& \left.\left.-\frac{\partial^{2}}{\partial J_{p_{2} p_{2}}^{a} \partial J_{p_{1} p_{1}}^{a}}\right)\left.\mathcal{Z}[J]\right|_{J=0}\right\} \text {. }
\end{aligned}
$$

The first line generates for $m \neq p_{1}$ and $m \neq p_{2}$ either a 4-point function with one boundary or two 2-point functions with one boundary, respectively. Functions with higher boundaries $B \geq 2$ appear in case of $m=p_{1}$ or $m=p_{2}$. All terms are found by comparing with the expansion (3.4).

We remind that in Proposition 2 correlation functions of genus $g \geq 1$ are also included. To see this one has to expand the correlation functions in a genus expansion. More information can be found in [12]. The Schwinger-Dyson equation of the 2-point function depends on $\lambda^{\prime 2}$, since graphs exist only with an even number of vertices.

Proposition 3 Let $N \geq 3$. The Schwinger-Dyson equation for the $N$-point function in the 3-colour matrix model with an external field $E$ is for pairwise different $p_{i}, p_{j}$ given by

$$
\begin{aligned}
& G_{\left|p_{1} \ldots p_{N}\right|}^{a_{1} \ldots a_{N}}=-\frac{\lambda^{\prime}}{\left(E_{p_{1}}^{2}-E_{p_{2}}^{2}\right)} \sum_{b=1}^{3}\left(\sigma_{a_{1} a_{N} b} G_{\left|p_{2} \ldots p_{N-1} p_{N}\right|}^{a_{2} \ldots a_{N-1} b}-\sigma_{a_{1} a_{2} b} G_{\left|p_{1} p_{3} p_{4} \ldots p_{N}\right|}^{b a_{3} a_{4} \ldots a_{N}}\right) \\
& -\frac{\lambda^{\prime 2}}{V^{2}\left(E_{p_{1}}^{2}-E_{p_{2}}^{2}\right)} \\
& \times\left\{V \left(\sum_{\substack{m=0 \\
m \neq p_{1}}}^{\mathcal{N}} \frac{G_{\left|m p_{2} \ldots p_{N}\right|}^{a_{1} a_{2} \ldots a_{N}}-G_{\left|p_{1} p_{2} \ldots p_{N}\right|}^{a_{1} a_{2} \ldots a_{N}}}{E_{p_{1}}-E_{m}}\right.\right. \\
& \left.-\sum_{\substack{m=0 \\
m \neq p_{2}}}^{\mathcal{N}} \frac{G_{\left|p_{1} m p_{3} \ldots p_{N}\right|}^{a_{1} a_{2} a_{3} \ldots a_{N}}-G_{\left|p_{1} p_{2} \ldots p_{N}\right|}^{a_{1} a_{2} \ldots a_{N}}}{E_{p_{2}}-E_{m}}\right) \\
& +\sum_{k=2}^{N}\left(\frac{G_{\left|p_{k} p_{2} \ldots p_{k-1}\right| p_{k} p_{k+1} \ldots p_{N} \mid}^{a_{1} a_{2} \ldots a_{k-1} \mid a_{k} a_{k+1} \ldots a_{N}}-G_{\left|p_{k} p_{2} \ldots p_{k-1}\right| p_{1} p_{k+1} \ldots p_{N} \mid}^{a_{1} a_{2} \ldots a_{k-1} \mid a_{k} a_{k+1} \ldots a_{N}}}{E_{p_{1}}-E_{p_{k}}}\right. \\
& \left.-\frac{G_{\left|p_{k+1} p_{3} \ldots p_{k}\right| p_{1} p_{k+1} \ldots p_{N} \mid}^{a_{2} a_{3} \ldots a_{k} \mid a_{1} a_{k+1} \ldots a_{N}}-G_{\left|p_{2} \ldots p_{k}\right| p_{1} p_{k+1} \ldots p_{N} \mid}^{a_{2} \ldots a_{k} \mid a_{1} a_{k+1} \ldots a_{N}}}{E_{p_{2}}-E_{p_{k+1}}}\right) \\
& +\sum_{k=3}^{N-1} V^{2}\left(G_{\left|p_{k} p_{2} \ldots p_{k-1}\right|}^{a_{1} a_{2} \ldots a_{k-1}} \frac{G_{\left|p_{k} \ldots p_{N}\right|}^{a_{k} \ldots a_{N}}-G_{\left|p_{1} p_{k+1} \ldots p_{N}\right|}^{a_{k} a_{k+1} \ldots a_{N}}}{E_{p_{1}}-E_{p_{k}}}\right. \\
& \left.-G_{\left|p_{1} p_{k+1} \ldots p_{N}\right|}^{a_{1} a_{k+1} \ldots a_{N}} \frac{G_{\left|p_{k+1} p_{3} \ldots p_{k}\right|}^{a_{2} a_{3} \ldots a_{k}}-G_{\left|p_{2} \ldots p_{k}\right|}^{a_{2} \ldots a_{k}}}{E_{p_{2}}-E_{p_{k+1}}}\right) \\
& +\sum_{b=1}^{3} \sum_{m=0}^{\mathcal{N}}\left(G_{\left|p_{1} m p_{1} \ldots p_{N}\right|}^{b b a_{1} \ldots a_{N}}-G_{\left|p_{1} p_{2} m p_{2} \ldots p_{N}\right|}^{a_{1} b b a_{2} \ldots a_{N}}\right. \\
& +\frac{1}{V}\left(G_{\left|p_{1} m\right| p_{1} \ldots p_{N} \mid}^{b b \mid a_{1} \ldots a_{N}}-G_{\left|p_{2} m\right| p_{1} \ldots p_{N} \mid}^{b b \mid a_{1} \ldots a_{N}}\right)
\end{aligned}
$$

$$
\begin{aligned}
& \left.+V G_{\left|p_{1} \ldots p_{N}\right|}^{a_{1} \ldots a_{N}}\left(G_{\left|p_{1} m\right|}^{b b}-G_{\left|p_{2} m\right|}^{b b}\right)\right) \\
& +\sum_{b=1}^{3} \sum_{k=2}^{N}\left(\frac { 1 } { V } \left(G_{\left|p_{k} p_{1} \ldots p_{k-1}\right| p_{1} p_{k} \ldots p_{N} \mid}^{b a_{1} \ldots a_{k-1} \mid b a_{k} \ldots a_{N}}\right.\right. \\
& \left.-G_{\left|p_{k+1} p_{2} \ldots p_{k}\right| p_{2} p_{k+1} \ldots p_{N} p_{1} \mid}^{b a_{2} \ldots a_{k} \mid b a_{k+1} \ldots a_{N} a_{1}}\right) \\
& +V\left(G_{\left|p_{k} p_{1} \ldots p_{k-1}\right|}^{b a_{1} \ldots a_{k-1}} G_{\left|p_{1} p_{k} \ldots p_{N}\right|}^{b a_{k} \ldots a_{N}}\right. \\
& \left.\left.-G_{\left|p_{k+1} p_{2} \ldots p_{k}\right|}^{b a_{2} \ldots a_{k}} G_{\left|p_{2} p_{k+1} \ldots p_{N} p_{1}\right|}^{b a_{k_{1}} \ldots a_{N} a_{1}}\right)\right) \\
& +\sum_{b=1}^{3}\left(\frac{1}{V^{2}}\left(G_{\left|p_{1}\right| p_{1}\left|p_{1} \ldots p_{N}\right|}^{b|b| a_{1} \ldots a_{N}}-G_{\left|p_{2}\right| p_{2}\left|p_{1} \ldots p_{N}\right|}^{b|b| a_{1} \ldots a_{N}}\right)\right. \\
& +\frac{1}{V}\left(G_{\left|p_{1}\right| p_{1} p_{1} \ldots p_{N}}^{b \mid b a_{1} \ldots a_{N}}-G_{\left|p_{2}\right| p_{2} p_{2} \ldots p_{N} p_{1}}^{b \mid b a_{2} \ldots a_{N} a_{1}}\right) \\
& \left.\left.+G_{\left|p_{1} \ldots p_{N}\right|}^{a_{1} \ldots a_{N}}\left(G_{\left|p_{1}\right| p_{1} \mid}^{b \mid b}-G_{\left|p_{2}\right| p_{2} \mid}^{b \mid b}\right)\right)\right\}
\end{aligned}
$$

where $p_{N+1} \equiv p_{1}$.

Proof We use the definition of the $N$-point function for pairwise different $p_{i}, p_{j}$. With the expression of the partition function (3.1), we obtain

$$
\begin{aligned}
& G_{\left|p_{1} \ldots p_{N}\right|}^{a_{1} \ldots a_{N}}=\left.\frac{1}{V} \frac{\partial^{N}}{\partial J_{p_{1} p_{2}}^{a_{1}} \ldots J_{p_{N} p_{1}}^{a_{N}}} \frac{\mathcal{Z}[J]}{\mathcal{Z}[0]}\right|_{J=0} \\
& =-\frac{\lambda^{\prime}}{H_{p_{1} p_{2}} V^{2} \mathcal{Z}[0]} \frac{\partial^{N-1}}{\partial J_{p_{2} p_{3}}^{a_{2}} \ldots J_{p_{N} p_{1}}^{a_{N}}} \sum_{b, c=1}^{3} \sigma_{a_{1} b c} \\
& \times\left.\sum_{n=0}^{\mathcal{N}} \frac{\partial^{2}}{\partial J_{p_{1} n}^{b} J_{n p_{2}}^{c}} \mathcal{Z}[J]\right|_{J=0} .
\end{aligned}
$$

Here the first derivative $\frac{\partial}{\partial J_{p_{1} p_{2}}^{a_{1}}}$ applied to $\mathcal{Z}_{\text {free }}[J]$ yields $\frac{V}{H_{p_{1} p_{2}}} J_{p_{2} p_{1}}^{a_{1}}$, which can only be differentiated by the interaction in $\mathcal{Z}[Z]$ because of $p_{3} \neq p_{1}$ and $p_{2} \neq p_{4}$. Applying Lemma 1 yields

$$
\begin{aligned}
= & -\frac{\lambda^{\prime}}{\left(E_{p_{1}}^{2}-E_{p_{2}}^{2}\right) V \mathcal{Z}[0]} \frac{\partial^{N-1}}{\partial J_{p_{2} p_{3}}^{a_{2}} \ldots J_{p_{N} p_{1}}^{a_{N}}} \\
\times & {\left[\sum_{b, c=1}^{3} \sigma_{a_{1} b c} \sum_{m=0}^{\mathcal{N}}\left(J_{m p_{1}}^{b} \frac{\partial}{\partial J_{m p_{2}}^{c}}-J_{p_{2} m}^{c} \frac{\partial}{\partial J_{p_{1} m}^{b}}\right)\right.} \\
& +\frac{\lambda^{\prime}}{V^{2}} \sum_{b=1}^{3}\left\{\sum _ { m = 0 } ^ { \mathcal { N } } \left(\frac{\partial^{3}}{\partial J_{p_{1} m}^{b} \partial J_{m p_{1}}^{b} \partial J_{p_{1} p_{2}}^{a_{1}}}\right.\right. \\
& \left.-\frac{\partial^{3}}{\partial J_{p_{1} p_{2}}^{a_{1}} \partial J_{p_{2} m}^{b} \partial J_{m p_{2}}^{b}}\right) \\
& +\sum_{\substack{\mathcal{N}_{m, n=0} \\
n \neq p_{1}}} \frac{V}{E_{p_{1}}-E_{n}} \frac{\partial}{\partial J_{n p_{2}}^{a_{1}}}\left(J_{m p_{1}}^{b} \frac{\partial}{\partial J_{m n}^{b}}-J_{n m}^{b} \frac{\partial}{\partial J_{p_{1} m}^{b}}\right)
\end{aligned}
$$




$$
\begin{aligned}
& -\sum_{\substack{m, n=0 \\
n \neq p_{2}}}^{\mathcal{N}} \frac{V}{E_{p_{2}}-E_{n}} \frac{\partial}{\partial J_{p_{1} n}^{a_{1}}}\left(J_{p_{2} m}^{b} \frac{\partial}{\partial J_{n m}^{b}}\right. \\
& \left.\left.\left.-J_{m n}^{b} \frac{\partial}{\partial J_{m p_{2}}^{b}}\right)\right\}\right]\left.\mathcal{Z}[J]\right|_{J=0} .
\end{aligned}
$$

The first term of (5.1a) contributes only for $b=a_{N}$ and $m=$ $p_{N}$ and the second term only for $c=a_{2}$ and $m=p_{3}$. This generates the term proportional to $\lambda^{\prime}$. Line $(5.1 \mathrm{~b})$ produces three different types of terms for arbitrary $m$, the $(2+N)$ point functions with $B=1$, the multiplication of 2-point with $N$-point functions, and $(2+N)$-point functions with $B=2$. If in (5.1b) $m=p_{k}$ for the first term with $2 \leq k \leq N$ (for the second term with $3 \leq k \leq N$ or $k=1$ ), additionally $(k+(N+2-k))$-point functions with $B=2$ and the multiplication of $k$-point with $(N+2-k)$-point functions with $B=1$ are generated. In case of $m=p_{1}$ for the left term ( $m=p_{2}$ for the right term) (5.1b) produces either $(1+1+N)$-point functions with $B=3,(1+(1+N))$-point functions with $B=2$ or the multiplication of $(1+1)$-point with $N$-point functions.

Finally, we look at (5.1c) and (5.1d) together. The first terms again contribute only for $b=a_{N}$ and $m=p_{N}$ in (5.1c) or for $b=a_{2}$ and $m=p_{3}$ in (5.1d). Since the sum over $n$ survives, $N$-point functions arise. If $n=p_{k}$ for $k \neq 1$ in (5.1c) and for $k \neq 2$ in (5.1d) one gets either $(k+(N-k))$ point functions or the multiplication of $k$-point functions with ( $N-k$ )-point functions with $B=1$. For the second term in (5.1c) and (5.1d) each derivative have to be taken into account. If the derivative in front of the brackets in $(5.1 \mathrm{c})$ and (5.1d) acts on $J_{n m}^{b}$ or $J_{m n}^{b}$, the sum over $n$ survives again and has a prefactor depending on $E_{n}$, but no $n$ appears in the $N$-point function. If any other derivative $\frac{\partial}{\partial J_{p_{k+1} a_{k+2}}^{a_{k+1}}}$, for some $k \geq 1$, acts on the second term, $n, m, b$ will be fixed and it will produces $N$-point functions, $(k+(N-k))$-point functions with $B=2$ and the multiplication of $k$-point with $(N-k)$-point functions. Collecting all and making use of (3.3) to get the correct prefactor in $V$, one find all the terms appearing in Proposition 3.

The first term shows that a $(N-1)$-point function only contributes for different adjacent colours, because of $\sigma_{a_{1} a_{N} b}$ and $\sigma_{a_{1} a_{2} b}$. This fact fits perfectly with a loop expansion. Furthermore, the 2-point function is assigned with a special rôle, since the sum over $m$ only appears for the $N$-point and 2-point function even in the large $\mathcal{N}, V$ limit.

It should be emphasised that not all combinations of the colours for the correlation functions are possible. The 2point function is of the form $G_{\left|p_{1} p_{2}\right|}^{a a}$ and the 3-point function $\sigma_{a b c} G_{\left|p_{1} p_{2} p_{3}\right|}^{a b c}$. There exists no 4-point function equipped with all three colours simultaneously, and so on. These prop- erties which are first recognized by loop expansion are intrinsically presented in the Schwinger-Dyson equations.

\subsection{Large $(\mathcal{N}, V)$-limit}

Sending $\mathcal{N}, V \rightarrow \infty$ with constant ratio $\frac{\mathcal{N}}{V}=\mu^{2} \Lambda^{2}$, the sum is turned into an integral by the transformation of the discrete elements to continuous variables $m \rightarrow V \mu^{2} q$

$\lim _{\substack{\mathcal{N}, V \rightarrow \infty \\ \mathcal{N}^{N}=\mu^{2} \Lambda^{2}}} \frac{1}{V} \sum_{m=0}^{\mathcal{N}} f\left(\frac{m}{V}\right)=\mu^{2} \int_{0}^{\Lambda^{2}} d q f\left(\mu^{2} q\right)$.

The eigenvalues of the external field are in the linear case given by $E_{m}=\mu^{2}\left(q+\frac{1}{2}\right)$. To get rid of the mass squared $\mu^{2}$, we redefine in the following way

$G_{p_{1} p_{2}}^{a a}:=\lim _{\mathcal{N}, V \rightarrow \infty} \mu^{2} G_{\left|p_{1} p_{2}\right|}^{a a}, \quad \lambda:=\frac{\lambda^{\prime}}{\mu^{2}}$.

An important fact is that in this limit only functions with genus $g=0$ survive [12]. Furthermore, we assume that all these functions of genus zero are $\mathcal{O}\left(V^{0}\right)$. The perturbation theory shows that in combination with the definition (3.3) this is the right assumption (see also $[14,15]$ ).

The equation of Proposition 2 breaks down to a closed equation, since 4-point functions and functions with $B \geq 2$ vanish. The $\operatorname{limit}_{\lim _{p_{2} \rightarrow p_{1}}} \frac{G_{n, p_{1} q}{ }^{a a}-G_{n, p_{2} q} a a}{p_{1}-p_{2}}$ is in perturbation theory well-defined, therefore this limit should also exist in the non-perturbative case. Sending $\Lambda^{2} \rightarrow \infty$ the closed integral equation for the 3 -colour model is obtained

$$
\begin{aligned}
G_{p_{1} p_{2}}^{a a}= & \frac{1}{1+p_{1}+p_{2}}+\frac{\lambda^{2}}{\left(1+p_{1}+p_{2}\right)\left(p_{1}-p_{2}\right)} \\
& \times\left(3 G_{p_{1} p_{2}}^{a a} \int_{0}^{\infty} d q\left(G_{q p_{2}}^{a a}-G_{p_{1} q}^{a a}\right)\right. \\
& \left.-\int_{0}^{\infty} d q \frac{G_{p_{1} q}^{a a}-G_{p_{1} p_{2}}^{a a}}{q-p_{2}}+\int_{0}^{\infty} d q \frac{G_{p_{2} q}^{a a}-G_{p_{1} p_{2}}^{a a}}{q-p_{1}}\right)
\end{aligned}
$$

We have assumed that $G_{p_{1} p_{2}}^{b b}$ does not depend directly on the colour $b$ so that $\sum_{b=1}^{3} G_{p_{1} p_{2}}^{b b}=3 G_{p_{1} p_{2}}^{a a}$.

\subsection{Perturbative solution}

Using the expansion (2.1) the closed integral equation (5.2) provides a recursive equation for $n \geq 1$ of the form

$$
\begin{aligned}
G_{2 n, p_{1} p_{2}}^{a a}= & \frac{1}{\left(1+p_{1}+p_{2}\right)\left(p_{1}-p_{2}\right)} \\
& \times\left(3 \sum_{i=0}^{n-1} G_{2(n-1-i), p_{1} p_{2}}^{a a} \int_{0}^{\infty} d q\left(G_{2 i, q p_{2}}^{a a}-G_{2 i, p_{1} q}^{a a}\right)\right.
\end{aligned}
$$




$$
\begin{aligned}
& -\int_{0}^{\infty} d q \frac{G_{2 n-2, p_{1} q}-G_{2 n-2, p_{1} p_{2}}}{q-p_{2}} \\
& \left.+\int_{0}^{\infty} d q \frac{G_{2 n-2, p_{2} q}-G_{2 n-2, p_{1} p_{2}}}{q-p_{1}}\right)
\end{aligned}
$$

and $G_{0, p_{1} p_{2}}^{a a}=\frac{1}{1+p_{1}+p_{2}}$. Equation (5.3) is linear which enables a very easy way to study this model in comparison to other noncommutative quantum field theory models. The convergence of loop expansion is hopeless, since the number of graphs of order $\lambda^{2 n}$ is at least of order $\mathcal{O}(n !)$, however the recursive equation gives directly the sum over all graphs of a certain order $\lambda^{2 n}$.

\section{Order $n=1$}

It is easy to verify with $\frac{\frac{1}{1+x+y}-\frac{1}{1+x+z}}{y-z}=-\frac{1}{(1+x+y)(1+x+z)}$ the result of (2.2) by

$$
\begin{aligned}
G_{2, p_{1} p_{2}}^{a a}= & \frac{1}{\left(1+p_{1}+p_{2}\right)\left(p_{1}-p_{2}\right)} \\
& \times\left(\frac{3}{1+p_{1}+p_{2}} \int_{0}^{\infty} d q\left(\frac{1}{1+p_{2}+q}-\frac{1}{1+p_{1}+q}\right)\right. \\
& \left.-\frac{1}{1+p_{1}+p_{2}} \int_{0}^{\infty} d q\left(\frac{1}{1+p_{2}+q}-\frac{1}{1+p_{1}+q}\right)\right) \\
= & \frac{2 \log \left(\frac{1+p_{1}}{1+p_{2}}\right)}{\left(1+p_{1}+p_{2}\right)^{2}\left(p_{1}-p_{2}\right)} .
\end{aligned}
$$

Order $n=2$

Inserting $G_{0, p_{1} p_{2}}^{a a}$ and $G_{2, p_{1} p_{2}}^{a a}$ into (5.3) to obtain

$$
\begin{aligned}
G_{4, p_{1} p_{2}}^{a a}= & \frac{6 \log \left(\frac{1+p_{1}}{1+p_{2}}\right)}{\left(1+p_{1}+p_{2}\right)^{2}\left(p_{1}-p_{2}\right)^{2}} \\
& \times \int_{0}^{\infty} d q \frac{p_{1}-p_{2}}{\left(1+p_{1}+q\right)\left(1+p_{2}+q\right)} \\
& +\frac{6}{\left(1+p_{1}+p_{2}\right)^{2}\left(p_{1}-p_{2}\right)} \\
& \times \int_{0}^{\infty} d q\left(\frac{\log \left(\frac{1+p_{2}}{1+q}\right)}{\left(1+q+p_{2}\right)^{2}\left(p_{2}-q\right)}\right. \\
& \left.-\frac{\log \left(\frac{1+p_{1}}{1+q}\right)}{\left(1+q+p_{1}\right)^{2}\left(p_{1}-q\right)}\right) \\
& -\frac{2}{\left(1+p_{1}+p_{2}\right)\left(p_{1}-p_{2}\right)} \\
& \times \int_{0}^{\infty} \frac{\log \left(\frac{1+p_{1}}{1+q}\right)}{\left(1+\frac{\left(1+q+p_{1}\right)^{2}\left(p_{1}-q\right)}{q}-\frac{1}{\left(1+p_{1}+p_{2}\right)^{2}\left(p_{1}-p_{2}\right)}\right.} \\
& \frac{2}{\left.1+p_{1}+p_{2}\right)\left(p_{1}-p_{2}\right)}
\end{aligned}
$$

$$
\times \int_{0}^{\infty} d q \frac{\frac{\log \left(\frac{1+q}{1+p_{2}}\right)}{\left(1+q+p_{2}\right)^{2}\left(q-p_{2}\right)}-\frac{\log \left(\frac{1+p_{1}}{1+p_{2}}\right)}{\left(1+p_{1}+p_{2}\right)^{2}\left(p_{1}-p_{2}\right)}}{q-p_{1}}
$$

(5.4a) is given by

$\frac{6 \log \left(\frac{1+p_{1}}{1+p_{2}}\right)^{2}}{(1+a+b)^{3}(a-b)^{2}}$

Using the definition of the dilogarithm

$\operatorname{Li}_{2}(-x)=-\int_{0}^{x} d u \frac{\log (1+u)}{u}$

(5.4b) is then determined by

$$
\begin{gathered}
\frac{6}{\left(1+p_{1}+p_{2}\right)^{2}\left(p_{1}-p_{2}\right)}\left(\frac{\log \left(1+p_{1}\right)}{p_{1}\left(1+p_{1}\right)\left(1+2 p_{1}\right)}\right. \\
+\frac{\log \left(1+p_{1}\right)^{2}+2 \operatorname{Li}_{2}\left(-p_{1}\right)-\frac{\pi^{2}}{6}}{\left(1+2 p_{1}\right)^{2}} \\
-\frac{\log \left(1+p_{2}\right)}{p_{2}\left(1+p_{2}\right)\left(1+2 p_{2}\right)} \\
\left.-\frac{\log \left(1+p_{2}\right)^{2}+2 \operatorname{Li}_{2}\left(-p_{2}\right)-\frac{\pi^{2}}{6}}{\left(1+2 p_{2}\right)^{2}}\right) .
\end{gathered}
$$

Lines $(5.4 \mathrm{c}+5.4 \mathrm{~d})$ are computed directly to avoid a singularity at $p_{1}=p_{2}$, and is given by

$$
\begin{aligned}
& \frac{2}{\left(1+p_{1}+p_{2}\right)^{2}\left(p_{1}-p_{2}\right)}\left(\frac{\log \left(1+p_{2}\right)}{p_{2}\left(1+2 p_{2}\right)\left(1+p_{2}\right)}\right. \\
& -\frac{\log \left(1+p_{1}\right)}{p_{1}\left(1+2 p_{1}\right)\left(1+p_{1}\right)} \\
& +\frac{\left(2+3 p_{1}+p_{2}\right)\left(\frac{\pi^{2}}{6}-\log \left(1+p_{1}\right)^{2}-2 \operatorname{Li}_{2}\left(-p_{1}\right)\right)}{\left(1+2 p_{1}\right)^{2}\left(1+p_{1}+p_{2}\right)} \\
& \left.-\frac{\left(2+3 p_{2}+p_{1}\right)\left(\frac{\pi^{2}}{6}-\log \left(1+p_{2}\right)^{2}-2 \operatorname{Li}_{2}\left(-p_{2}\right)\right)}{\left(1+2 p_{2}\right)^{2}\left(1+p_{1}+p_{2}\right)}\right)
\end{aligned}
$$

Adding all terms and using the well known identity

$\mathrm{Li}_{2}(-x)+\frac{1}{2} \log (1+x)^{2}=-\mathrm{Li}_{2}\left(\frac{x}{1+x}\right)$,

the result is then given by

$$
\begin{aligned}
G_{4, p_{1} p_{2}}^{a a}= & \frac{2}{\left(1+p_{1}+p_{2}\right)^{2}\left(p_{1}-p_{2}\right)} \\
& \times\left(\frac{3 \log \left(\frac{1+p_{1}}{1+p_{2}}\right)^{2}}{\left(1+p_{1}+p_{2}\right)\left(p_{1}-p_{2}\right)}\right.
\end{aligned}
$$




$$
\begin{aligned}
& +\frac{2 \log \left(1+p_{1}\right)}{p_{1}\left(1+2 p_{1}\right)\left(1+p_{1}\right)}-\frac{2 \log \left(1+p_{2}\right)}{p_{2}\left(1+2 p_{2}\right)\left(1+p_{2}\right)} \\
& -\frac{\left(1+2 p_{2}\right)\left(\frac{\pi^{2}}{6}+2 \mathrm{Li}_{2}\left(\frac{p_{1}}{1+p_{1}}\right)\right)}{\left(1+2 p_{1}\right)^{2}\left(1+p_{1}+p_{2}\right)} \\
& \left.+\frac{\left(1+2 p_{1}\right)\left(\frac{\pi^{2}}{6}+2 \mathrm{Li}_{2}\left(\frac{p_{2}}{1+p_{2}}\right)\right)}{\left(1+2 p_{2}\right)^{2}\left(1+p_{1}+p_{2}\right)}\right)
\end{aligned}
$$

Equation (5.6) is confirmed by loop expansion in Appendix A.

Order $n=3$

The 2-point functions $G_{0, p_{1} p_{2}}^{a a}, G_{2, p_{1} p_{2}}^{a a}$ and $G_{4, p_{1} p_{2}}^{a a}$ are inserted into (5.3). We split the integrals into individual parts, which certainly converge. The identity (5.5) is used to achieve terms of the form $\operatorname{Li}_{2}(-x)$, which are easier to integrate. With the definition of the trilogarithm

$\operatorname{Li}_{3}(-x)=\int_{0}^{x} d u \frac{\operatorname{Li}_{2}(-u)}{u}$,

and the identities

$$
\begin{aligned}
\operatorname{Li}_{3}(-x)= & \operatorname{Li}_{3}\left(-\frac{1}{x}\right)-\frac{1}{6} \log (x)^{3}-\frac{\pi^{2}}{6} \log (x) \\
\operatorname{Li}_{3}(-x)= & -\operatorname{Li}_{3}\left(\frac{x}{1+x}\right)+\frac{\log (1+x)^{3}}{3}-\frac{\log (x) \log (1+x)^{2}}{2} \\
& -\frac{\pi^{2} \log (1+x)}{6}+\zeta(3),
\end{aligned}
$$

where $\zeta(x)$ is the Riemannian $\zeta$ function, we finally find the correlation function of order $\lambda^{6}$

$$
\begin{aligned}
G_{6, p_{1} p_{2}}^{a a}= & \left\{\log \left(1+p_{1}\right) f_{1}\left(p_{1}, p_{2}\right)\right. \\
& +\pi^{2} \log \left(1+p_{1}\right) f_{2}\left(p_{1}, p_{2}\right) \\
& +\log \left(1+p_{1}\right)^{2} f_{3}\left(p_{1}, p_{2}\right) \\
& +\left(\operatorname{Li}_{2}\left(\frac{p_{1}}{1+p_{1}}\right)+\frac{\pi^{2}}{6}\right) f_{4}\left(p_{1}, p_{2}\right) \\
& +\operatorname{Li}_{2}\left(\frac{p_{1}}{1+p_{1}}\right) \log \left(\frac{1+p_{1}}{1+p_{2}}\right) f_{5}\left(p_{1}, p_{2}\right) \\
& +\left(\operatorname{Li}_{3}\left(-p_{1}\right)+\operatorname{Li} 3\left(\frac{p_{1}}{1+p_{1}}\right)\right. \\
& +\operatorname{Li}_{2}\left(\frac{p_{1}}{1+p_{1}}\right) \log \left(1+p_{1}\right) \\
& \left.+\frac{\log \left(1+p_{1}\right)^{3}}{6}-\frac{\pi^{2} \log \left(1+p_{1}\right)}{6}\right) f_{6}\left(p_{1}, p_{2}\right) \\
& \left.+\left(\operatorname{Li} 3\left(\frac{p_{1}}{1+p_{1}}\right)+\frac{\pi^{2} \log \left(1+p_{1}\right)}{3}\right) f_{7}\left(p_{1}, p_{2}\right)\right\} \\
& +\left\{p_{1} \leftrightarrow p_{2}\right\} \\
& +\log \left(\frac{1+p_{1}}{1+p_{2}}\right)^{3} f_{8}\left(p_{1}, p_{2}\right) \\
& +\log \left(1+p_{1}\right) \log \left(1+p_{2}\right) f_{9}\left(p_{1}, p_{2}\right) \\
& +\pi^{2} f_{10}\left(p_{1}, p_{2}\right) \\
& +\pi^{2} \log (2) f_{11}\left(p_{1}, p_{2}\right)+\zeta(3) f_{12}\left(p_{1}, p_{2}\right)
\end{aligned}
$$

with

$$
\begin{aligned}
& f_{1}\left(p_{1}, p_{2}\right)=-\frac{8}{p_{1}\left(1+p_{1}\right)^{2}\left(1+2 p_{1}\right)^{2}\left(p_{1}-p_{2}\right)\left(1+p_{1}+p_{2}\right)^{2}} \\
& f_{2}\left(p_{1}, p_{2}\right)=\frac{4\left\{\left(p_{1}-p_{2}\right)^{3}+\left(p_{1}+p_{2}+1\right)\left(7\left(p_{1}+p_{2}+1\right)^{2}-3\left(2 p_{2}+1\right)\left(p_{1}-p_{2}\right)\right)\right\}}{\left(1+2 p_{1}\right)^{3}\left(p_{1}-p_{2}\right)\left(1+p_{1}+p_{2}\right)^{4}\left(1+2 p_{2}\right)^{2}} \\
& f_{3}\left(p_{1}, p_{2}\right)=\frac{6}{p_{1}^{2}\left(1+p_{1}\right)^{2}\left(1+2 p_{1}\right)^{3}\left(p_{1}-p_{2}\right)^{2}\left(1+p_{1}+p_{2}\right)^{3}} \\
& \times\left\{( 1 + p _ { 1 } + p _ { 2 } ) \left(2\left(p_{1}-p_{2}\right)\left(1+10 p_{1}\left(1+p_{1}\right)\right)\right.\right. \\
& \left.+3\left(1+p_{1}\right)\left(1+2 p_{1}\right)\right) \\
& \left.+2 p_{1}\left(1+p_{1}\right)\left(1+2 p_{1}\right)^{2}\right\} \\
& f_{4}\left(p_{1}, p_{2}\right)=-\frac{4}{p_{1}\left(1+p_{1}\right)^{2}\left(1+2 p_{1}\right)^{3}\left(p_{1}-p_{2}\right)^{2}\left(1+p_{1}+p_{2}\right)^{3}} \\
& \times\left\{( 1 + p _ { 1 } + p _ { 2 } ) \left(2\left(1+p_{2}\right)+p_{1}\left(11+43 p_{1}\right.\right.\right. \\
& \left.\left.+38 p_{1}^{2}-6\left(3+4 p_{1}\right) p_{2}\right)\right) \\
& \left.-2\left(1+p_{1}\right)\left(1+2 p_{1}\right)^{3}\right\} \\
& f_{5}\left(p_{1}, p_{2}\right)=\frac{12\left\{2\left(p_{1}-p_{2}\right)^{2}+\left(2 p_{2}+1\right)\left(p_{1}+p_{2}+1\right)\right\}}{\left(1+2 p_{1}\right)^{2}\left(p_{1}-p_{2}\right)^{3}\left(1+p_{1}+p_{2}\right)^{4}} \\
& f_{6}\left(p_{1}, p_{2}\right)=-\frac{24\left\{\left(1+p_{1}+p_{2}\right)\left(10\left(p_{1}-p_{2}\right)^{2}+\left(1+3 p_{1}-p_{2}\right)^{2}\right)-\left(p_{1}-p_{2}\right)^{3}\right\}}{\left(1+2 p_{1}\right)^{4}\left(p_{1}-p_{2}\right)^{3}\left(1+p_{1}+p_{2}\right)^{3}} \\
& f_{7}\left(p_{1}, p_{2}\right)=-\frac{12\left\{5+6 p_{1}+4 p_{2}\right\}}{\left(1+2 p_{1}\right)^{4}\left(p_{1}-p_{2}\right)\left(1+p_{1}+p_{2}\right)^{3}} \\
& f_{8}\left(p_{1}, p_{2}\right)=\frac{20}{\left(1+p_{1}+p_{2}\right)^{4}\left(p_{1}-p_{2}\right)^{3}} \\
& f_{9}\left(p_{1}, p_{2}\right)=-\frac{24\left\{2 p_{1}^{2}-2 p_{1} p_{2}+p_{1}+2 p_{2}^{2}+p_{2}\right\}}{p_{1}\left(1+p_{1}\right)\left(1+2 p_{1}\right)\left(p_{1}-p_{2}\right)^{2} p_{2}\left(1+p_{2}\right)\left(1+2 p_{2}\right)\left(1+p_{1}+p_{2}\right)^{2}} \\
& f_{10}\left(p_{1}, p_{2}\right)=\frac{4}{3 p_{1}\left(1+p_{1}\right)\left(1+2 p_{1}\right)^{3} p_{2}\left(1+p_{2}\right)\left(1+2 p_{2}\right)^{3}\left(1+p_{1}+p_{2}\right)^{3}} \\
& \times\left[p _ { 1 } p _ { 2 } \left\{( p _ { 1 } + p _ { 2 } + 1 ) \left(48 p_{1}^{3}\right.\right.\right. \\
& +\left(-48 p_{1}^{2}-24 p_{1}+72\right) p_{2}^{2}+\left(-40 p_{1}^{2}-12 p_{1}+56\right) p_{2} \\
& \left.+88 p_{1}^{2}+56 p_{1}+32 p_{2}^{3}+24\right) \\
& \left.-\left(2 p_{1}+1\right)^{2}\left(4 p_{1}\left(p_{1}+1\right)-1\right)\right\} \\
& +2\left(2 p_{1}+1\right)^{2}\left(2 p_{2}+1\right)^{2}\left(p_{1}+p_{2}+1\right)^{3} \\
& \left.+p_{1}\left(p_{1}+1\right)\left(2 p_{1}+1\right)^{2}+p_{2}\left(p_{2}+1\right)\left(2 p_{2}+1\right)^{2}\right] \\
& f_{11}\left(p_{1}, p_{2}\right)=-\frac{32\left\{9\left(p_{1}-p_{2}\right)^{2}+7\left(p_{1}+p_{2}+1\right)^{2}\right\}}{\left(1+2 p_{1}\right)^{4}\left(1+2 p_{2}\right)^{4}\left(1+p_{1}+p_{2}\right)} \\
& f_{12}\left(p_{1}, p_{2}\right)=\frac{24\left\{\left(p_{1}-p_{2}\right)^{2}+5\left(p_{1}+p_{2}+1\right)^{2}\right\}}{\left(1+2 p_{1}\right)^{3}\left(1+2 p_{2}\right)^{3}\left(1+p_{1}+p_{2}\right)^{3}},
\end{aligned}
$$

where $\left\{p_{1} \leftrightarrow p_{2}\right\}$ are the first seven terms by interchanging $p_{1}$ and $p_{2}$. To obtain these results we computed a primitive of the integrals using a computer algebra system and took the limits $q \rightarrow 0$ and $q \rightarrow \infty$ by hand. More than 20 different type of loops contribute at sixth order in $\lambda$, and (5.7) is the sum of all of them.

Most of the terms in (5.7) are individually divergent in the limit $p_{2} \rightarrow p_{1}$ or $p_{1 / 2} \rightarrow 0$. However, in both limits $G_{6, p_{1} p_{2}}^{a a}$ has a finite result. For $p=p_{1}=p_{2}$ we find

$$
\begin{aligned}
G_{6, p p}^{a a}= & \frac{1776}{(1+2 p)^{7}}\left\{\operatorname{Li}_{3}(-p)+\frac{93}{74} \operatorname{Li}_{3}\left(\frac{p}{p+1}\right)\right. \\
& +\operatorname{Li}_{2}\left(\frac{p}{p+1}\right) \log (p+1) \\
& +\frac{1}{6} \log ^{3}(p+1)-\frac{14}{111} \pi^{2} \log (p+1)
\end{aligned}
$$




$$
\begin{aligned}
& \left.-\frac{14}{111} \pi^{2} \log (2)+\frac{5}{74} \zeta(3)\right\} \\
& +\frac{2 \pi^{2}(10 p(p(4 p+39)+60)+257)}{3(1+p)^{3}(1+2 p)^{6}} \\
& +\frac{2(9+10 p)}{p(1+2 p)^{7}}+\frac{4 \log (1+p)(5+7 p)}{p^{2}(1+p)^{2}(1+2 p)^{5}} \\
& -\frac{2 \log (1+p)^{2}(p(p+1)(546 p(p+1)+125)+11)}{p^{3}(1+p)^{3}(1+2 p)^{6}} \\
& +\frac{4 \operatorname{Li}_{2}\left(\frac{p}{1+p}\right)\left(7+(1+p)\left(176 p^{3}+75 p^{2}-44 p-11\right)\right)}{p^{2}(1+p)^{3}(1+2 p)^{6}}
\end{aligned}
$$

It is nice to see how in (5.8) the linear divergence for $p \rightarrow 0$ in the last four terms cancels perfectly, since $\lim _{p \rightarrow 0} \frac{\operatorname{Li}_{2}\left(\frac{p}{1+p}\right)}{p^{2}}=\frac{1}{p}+\mathcal{O}(1)$. Also remarkable to assess is the fact that all kind of functions appearing the first time at order $\lambda^{6}$ [first two lines of (5.8)] have the same dependence of $p$ in the denominator.

Sending $p_{2} \rightarrow p_{1}$ in (5.2) an integral equation with derivatives $\frac{\partial G_{p_{1} q}^{a a}}{\partial p_{1}}$ appears. Making use of all results (2.2),(5.6) and (5.7), the numerical solution for the 2-point function with zero momenta can be given up to the eighth-order

$$
\begin{aligned}
G_{00}^{a a}= & 1+2 \lambda^{2}+2\left(\pi^{2}-6\right) \lambda^{4} \\
& +\left\{\pi^{2}\left(\frac{514}{3}-224 \log (2)\right)+120 \zeta(3)-266\right\} \lambda^{6} \\
& +194.612 \lambda^{8}+\mathcal{O}\left(\lambda^{10}\right) .
\end{aligned}
$$

\section{Conclusion and outlook}

We have introduced the noncommutative 3-colour model as a quantum field theoretical model in two dimensions. We derived the Schwinger-Dyson equations of the 2-point function and the $N$-point functions for a single boundary component. This required a generalisation of the Ward-Takahashi identity to coloured models. This new identity seems to be related to a mixing symmetry between two colours. In the large $\mathcal{N}, V$ limit a closed integral equation (5.2) occurs, which is a non-perturbative result. This equation was used to find perturbative solutions up to the sixth order in the coupling constant.

The main aim for the future is to find an exact solution of (5.2) or to prove existence, if possible also uniqueness, of a solution. Furthermore, we want to extend this work to determine Schwinger-Dyson equations for $B \geq 2$, where already problems arise for the $(1+1)$-point function. Finally, we would like to treat the renormalisation problems in dimension 4 and 6.

Acknowledgements This work was supported by the Deutsche Forschungsgemeinschaft (SFB 878). A. H. wants to thank Jins de Jong for helpful discussions.
Open Access This article is distributed under the terms of the Creative Commons Attribution 4.0 International License (http://creativecomm ons.org/licenses/by/4.0/), which permits unrestricted use, distribution, and reproduction in any medium, provided you give appropriate credit to the original author(s) and the source, provide a link to the Creative Commons license, and indicate if changes were made.

Funded by SCOAP ${ }^{3}$.

\section{A. $G_{4, p_{1} p_{2}}^{a a}$ computation by graphs}

Representatives of all graphs with one boundary component and two external edges at the fourth order in $\lambda$ are the following graphs:
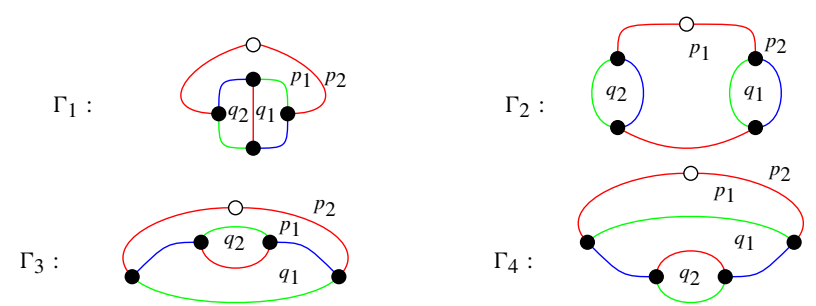

Let $a=a_{1}^{1}=a_{2}^{1}$. With straightforward computation by using the introduced rules in Sect. 2 one finds

$$
\begin{aligned}
& \tilde{G}_{p_{1} p_{2}}^{a a}\left(\Gamma_{1}\right)=\frac{\lambda^{4}}{\left(1+p_{1}+p_{2}\right)^{2}} \\
& \times \int_{0}^{\infty} \frac{\frac{d q_{1} d q_{2}}{1+q_{1}+q_{2}}}{\left(1+p_{1}+q_{1}\right)\left(1+p_{1}+q_{2}\right)\left(1+p_{2}+q_{1}\right)\left(1+p_{2}+q_{2}\right)} \\
& =\frac{\lambda^{4}}{\left(1+p_{1}+p_{2}\right)^{2}}\left(-\frac{\log \left(1+p_{1}\right)^{2}}{\left(p_{1}-p_{2}\right)^{2}\left(1+2 p_{1}\right)}\right. \\
& -\frac{\log \left(1+p_{2}\right)^{2}}{\left(p_{1}-p_{2}\right)^{2}\left(1+2 p_{2}\right)} \\
& -\frac{\pi^{2} / 6-2 \mathrm{Li}_{2}\left(-p_{1}\right)}{\left(1+2 p_{1}\right)\left(p_{1}-p_{2}\right)\left(1+p_{1}+p_{2}\right)} \\
& +\frac{\pi^{2} / 6-2 \operatorname{Li}_{2}\left(-p_{2}\right)}{\left(1+2 p_{2}\right)\left(p_{1}-p_{2}\right)\left(1+p_{1}+p_{2}\right)} \\
& \left.+\frac{2 \log \left(1+p_{1}\right) \log \left(1+p_{2}\right)}{\left(p_{1}-p_{2}\right)^{2}\left(1+p_{1}+p_{2}\right)}\right) \\
& \tilde{G}_{p_{1} p_{2}}^{a a}\left(\Gamma_{2}\right)=\frac{\lambda^{4}}{\left(1+p_{1}+p_{2}\right)^{3}} \\
& \times \int_{0}^{\infty} \frac{d q_{1} d q_{2}}{\left(1+p_{1}+q_{1}\right)\left(1+p_{2}+q_{1}\right)\left(1+p_{2}+q_{1}\right)\left(1+p_{2}+q_{2}\right)} \\
& =\frac{\lambda^{4}}{\left(1+p_{1}+p_{2}\right)^{3}} \frac{\log \left(\frac{1+p_{1}}{1+p_{2}}\right)^{2}}{\left(p_{1}-p_{2}\right)^{2}} \\
& \tilde{G}_{p_{1} p_{2}}^{a a}\left(\Gamma_{3}\right)=\frac{\lambda^{4}}{\left(1+p_{1}+p_{2}\right)^{2}} \\
& \times \int_{0}^{\infty} \frac{\frac{d q_{1} d q_{2}}{1+q_{1}+q_{2}}}{\left(1+p_{1}+q_{1}\right)^{2}\left(1+p_{1}+q_{2}\right)\left(1+p_{2}+q_{1}\right)} \\
& =\frac{\lambda^{4}}{\left(1+p_{1}+p_{2}\right)^{2}}\left(-\frac{\operatorname{Li}_{2}\left(-p_{1}\right)}{\left(1+2 p_{1}\right)^{2}\left(1+p_{1}+p_{2}\right)}\right. \\
& -\frac{\frac{\pi^{2}}{6}-\log \left(1+p_{1}\right)^{2}-\mathrm{Li}_{2}\left(-p_{1}\right)}{\left(1+2 p_{1}\right)\left(p_{1}-p_{2}\right)^{2}} \\
& -\frac{\frac{\pi^{2}}{6}-\log \left(1+p_{1}\right)^{2}-\mathrm{Li}_{2}\left(-p_{1}\right)}{\left(1+2 p_{1}\right)^{2}\left(p_{1}-p_{2}\right)}
\end{aligned}
$$




$$
\begin{aligned}
& +\frac{\frac{\pi^{2}}{6}-\log \left(1+p_{2}\right) \log \left(1+p_{1}\right)-\mathrm{Li}_{2}\left(-p_{2}\right)}{\left(1+p_{1}+p_{2}\right)\left(p_{1}-p_{2}\right)^{2}} \\
& \left.+\frac{\log \left(1+p_{1}\right)}{p_{1}\left(1+p_{1}\right)\left(1+2 p_{1}\right)\left(p_{1}-p_{2}\right)}\right) \\
\tilde{G}_{p_{1} p_{2}}^{a a}\left(\Gamma_{4}\right)= & \frac{\lambda^{4}}{\left(1+p_{1}+p_{2}\right)^{2}} \\
& \int_{0}^{\infty} \frac{\frac{d q_{1} d q_{2}}{1+q_{1}+q_{2}}}{\left(1+p_{1}+q_{1}\right)\left(1+p_{2}+q_{1}\right)^{2}\left(1+p_{2}+q_{2}\right)} \\
= & \frac{\lambda^{4}}{\left(1+p_{1}+p_{2}\right)^{2}}\left(-\frac{\mathrm{Li}_{2}\left(-p_{2}\right)}{\left(1+2 p_{2}\right)^{2}\left(1+p_{1}+p_{2}\right)}\right. \\
& -\frac{\frac{\pi^{2}}{6}-\log \left(1+p_{2}\right)^{2}-\mathrm{Li}_{2}\left(-p_{2}\right)}{\left(1+2 p_{2}\right)\left(p_{1}-p_{2}\right)^{2}} \\
& +\frac{\frac{\pi^{2}}{6}-\log \left(1+p_{2}\right)^{2}-\mathrm{Li}_{2}\left(-p_{2}\right)}{\left(1+2 p_{2}\right)^{2}\left(p_{1}-p_{2}\right)} \\
& +\frac{\frac{\pi^{2}}{6}-\log \left(1+p_{2}\right) \log \left(1+p_{1}\right)-\mathrm{Li}_{2}\left(-p_{1}\right)}{\left(1+p_{1}+p_{2}\right)\left(p_{1}-p_{2}\right)^{2}} \\
& \left.-\frac{\log \left(1+p_{2}\right)}{p_{2}\left(1+p_{2}\right)\left(1+2 p_{2}\right)\left(p_{1}-p_{2}\right)}\right) .
\end{aligned}
$$

We verify easily that $s\left(\Gamma_{1}\right)=2, s\left(\Gamma_{2}\right)=4, s\left(\Gamma_{3}\right)=4$ and $s\left(\Gamma_{4}\right)=4$. The correlation function of order $\lambda^{4}$ is finally given with identity (5.5) by

$$
\begin{aligned}
G_{4, p_{1} p_{2}}^{a a}= & \sum_{i=1}^{4} s\left(\Gamma_{i}\right) \tilde{G}_{p_{1} p_{2}}^{a a}\left(\Gamma_{i}\right) \\
= & \frac{2}{\left(1+p_{1}+p_{2}\right)^{2}\left(p_{1}-p_{2}\right)} \\
& \times\left(\frac{3 \log \left(\frac{1+p_{1}}{1+p_{2}}\right)^{2}}{\left(1+p_{1}+p_{2}\right)\left(p_{1}-p_{2}\right)}\right. \\
& +\frac{2 \log \left(1+p_{1}\right)}{p_{1}\left(1+2 p_{1}\right)\left(1+p_{1}\right)}-\frac{2 \log \left(1+p_{2}\right)}{p_{2}\left(1+2 p_{2}\right)\left(1+p_{2}\right)} \\
& -\frac{\left(1+2 p_{2}\right)\left(\frac{\pi^{2}}{6}+2 \operatorname{Li}_{2}\left(\frac{p_{1}}{1+p_{1}}\right)\right)}{\left(1+2 p_{1}\right)^{2}\left(1+p_{1}+p_{2}\right)} \\
& \left.+\frac{\left(1+2 p_{1}\right)\left(\frac{\pi^{2}}{6}+2 \operatorname{Li}_{2}\left(\frac{p_{2}}{1+p_{2}}\right)\right)}{\left(1+2 p_{2}\right)^{2}\left(1+p_{1}+p_{2}\right)}\right) .
\end{aligned}
$$

\section{References}

1. R.J. Baxter, Colorings of a hexagonal lattice. J. Math. Phys. 11, 784 (1970)

2. B. Eynard, C. Kristjansen, An iterative solution of the three-colour problem on a random lattice. Nucl. Phys. B 516, 529 (1998)

3. I.K. Kostov, Exact solution of the three-color problem on a random lattice. Phys. Lett. B 549, 245 (2002)

4. M.R. Douglas, S.H. Shenker, Strings in less than one dimension. Nucl. Phys. B 335, 635 (1990)

5. D.J. Gross, A.A. Migdal, Nonperturbative two-dimensional quantum gravity. Phys. Rev. Lett. 64, 127 (1990)

6. M. Kontsevich, Intersection theory on the moduli space of curves and the matrix airy function. Commun. Math. Phys. 147, 1 (1992)

7. E. Witten, Two-dimensional gravity and intersection theory on moduli space. Surv. Differ. Geom. 1, 243 (1990)

8. Y. Makeenko, G.W. Semenoff, Properties of hermitian matrix models in an external field. Mod. Phys. Lett. A 6, 3455 (1991)

9. E. Langmann, R.J. Szabo, K. Zarembo, Exact solution of quantum field theory on noncommutative phase spaces. J. High Energy Phys. 1, 17 (2004)

10. H. Grosse, H. Steinacker, Renormalization of the noncommutative $\phi^{3}$ model through the Kontsevich model. Nucl. Phys. B 746, 202 (2006)

11. H. Grosse, R. Wulkenhaar, Renormalisation of $\phi^{4}$-theory on noncommutative $\mathbb{R}^{4}$ in the matrix base. Commun. Math. Phys. 256, 305 (2005)

12. H. Grosse, R. Wulkenhaar, Self-dual noncommutative $\phi^{4}$-theory in four dimensions is a non-perturbatively solvable and non-trivial quantum field theory. Commun. Math. Phys. 329, 1069 (2014)

13. M. Disertori, R. Gurau, J. Magnen, V. Rivasseau, Vanishing of beta function of non-commutative $\Phi_{4}^{4}$ theory to all orders. Phys. Lett. B 649, 95 (2007)

14. H. Grosse, A. Sako, R. Wulkenhaar, Exact solution of matricial $\phi_{2}^{3}$ quantum field theory. Nucl. Phys. B 925, 319 (2017)

15. H. Grosse, A. Sako, R. Wulkenhaar, The $\phi_{4}^{3}$ and $\phi_{6}^{3}$ matricial QFT models have reflection positive two-point function. Nucl. Phys. B 926, 20 (2018)

16. H. Grosse, R. Wulkenhaar, Renormalisation of $\phi^{4}$-theory on noncommutative $\mathbb{R}^{2}$ in the matrix base. J. High Energy Phys. 12, 19 (2003) 